\title{
The Expression of Preaxial Polydactyly Is Influenced by Modifying Genetic Elements and Is Not Maintained by Chromosomal Inversion in an Avian Biomedical Model
}

\author{
E.A. Robb M.E. Delany \\ Department of Animal Science, University of California, Davis, Calif., USA
}

\section{Key Words}

Biomedical model $\cdot$ Breeding strategies $\cdot$ Chicken .

Epistasis · Fine-mapping · FISH • Inversion - LMBR1 •

Preaxial polydactyly $\cdot$ Recombination $\cdot$ Silkie breed $\cdot$ SNP • ZRS

\section{Abstract \\ Polydactyly (Po) is a common mutation found in many ver- tebrates. The UCD-Po.003 congenic chicken line was previ- ously characterized for Po inheritance (autosomal dominant) and the mutation was mapped to chromosome $2 p$. Here, we describe for the first time the range and variability of the phenotype in this congenic line. Further, we studied the hy- pothesis that a chromosomal inversion was responsible for the maintenance of a large $(6.3 \mathrm{Mb})$ candidate gene region. Fluorescence in situ hybridization employing BACs encom- passing a 10.7-Mb region of GGA2p showed that the Po chro- mosome was normal, i.e. exhibits the wild-type BAC order. Continued fine-mapping along with a change in breeding strategy reduced the size of the causative region to $1.43 \mathrm{Mb}$. Recent research indicates that the cause of preaxial Po re- sides within a 794-bp highly conserved zone of polarizing activity regulatory sequence (ZRS) element located in intron}

\section{KARGER}

Fax +4161306 1234

E-Mail karger@karger.ch

www.karger.com
(C) 2012 S. Karger AG, Basel

1424-8581/12/1361-0050\$38.00/0

Accessible online at:

www.karger.com/cgr
5 of the $L M B R 1$ gene; however, the ZRS polymorphism of interest is found in some but not all breeds of polydactylous chicken. Therefore, we sequenced the ZRS in 101 heterozygous and 30 unaffected (wild-type) individuals to establish the relevance of this region to the Po condition in the UCD-Po.003 congenic line. A single point mutation (C/A at coordinate GGA2p: 8,414,121) within the ZRS segregated with carrier status. The polydactylous UCD-Silkie line also maintains this SNP in addition to a single base deletion. An inheritance analysis of the phenotypic variation in UCDPo.003 suggests recessive epistasis as the mode of inheritance for the additional modifying genetic elements, residing outside the ZRS, to impact the preaxial polydactyl phenotype. These results contribute to our understanding of the cause of Po in an important vertebrate model.

Copyright $\odot 2012$ S. Karger AG, Basel

Polydactyly (Po), the addition of one or more extra digits, occurs in human as well as a variety of vertebrates including model organisms, domesticated animals, and livestock species. Such animals include, but are not limited to mouse, rat, chicken, Xenopus, cat, cow, dog, horse, pig, and sheep [Struthers, 1893; Danforth, 1947; Chase, 
1951; Uehlinger, 1969; Kren, 1975; Dennis and Leipold, 1979; Johnson et al., 1981; Pitel et al., 2000; Park et al., 2008; Gorbach et al., 2010; Biesecker, 2011]. This common malformation was one of the earliest genetic mutations studied in chicken, dating back to Punnett and colleagues in the 1920s [Bond, 1920, 1926; Punnett and Pease, 1929; Warren, 1944]. Po has been reported in a variety of bird species ranging from mallard and common loon to common swift and Anna's hummingbird, although it is inherently difficult to establish frequency of occurrence in the wild [Sakai, 2006 and references therein]. However, Po is a phenotype found in a number of domestic chicken breeds including Favorelle, Dorking, Houdan, Silkie, and Sultan [The American Poultry Association, 1998]. Pitel et al. [2000] mapped the dominant form of Po, showing incomplete penetrance, to GGA2p using microsatellite markers; this research allowed for the connection between the classical genetic map and the molecular genetic map. In the UCD-Po.003 congenic inbred line, the polydactylous phenotype is inherited in an autosomal dominant fashion and is similarly linked to GGA2p [Robb et al., 2011]. Within the UCD-Po.003 line, polydactyl expression is variable as homozygous (Po.003/Po.003) and heterozygous $(P o .003 /+)$ mutants may have an additional preaxial digit (single-digit duplication) on one or both feet [Robb et al., 2011], in 1 of 2 digit patterns, in addition to a possible preaxial digit on one, none, or both wings.

In human, the rate of expression varies and prevalence, which is dependent upon sex and race, ranges from 1.2 to 22.5 per 1,000 individuals [Everman, 2006]. Similar to chicken, the human polydactyl phenotype is variable, even within the same family [Everman, 2006]. Human Po can be inherited in an autosomal dominant fashion and has been associated with the $S H H$ and LMBR1 genes located on human chromosome 7q36.3, which is syntenic to the region of interest (GGA2p) for the UCD-Po.003 mutation.

Studies in mice on the role of $\mathrm{SHH}$ revealed that it is a key regulator in defining the limb anterior-posterior axis in early embryogenesis [Lettice et al., 2002, 2003] and is expressed in the mesenchyme along the posterior margin of the limb in a region called zone of polarizing activity (ZPA). Knockout $S h h^{-/-}$mice display 1 distinct digit on each hindlimb and a distal cartilaginous element on the forelimbs, albeit with severely truncated and fused bones in the fore- and hindlimbs [Chiang et al., 2001; Kraus et al., 2001]. In contrast, overexpression of SHH results in a variety of phenotypes including craniofacial defects and other skeletal anomalies, but does not cause Po [Riddle et al., 1993; Oro et al., 1997].

Genomics of Avian Polydactyly
Mouse knockout studies of various Lmbrl exons indicate that $L m b r l$ is not the direct cause of the Po mutation. Upon further assessment of the Lmbrl genetic region, it was determined that a ZPA regulatory sequence (ZRS), located within intron 5 , is the source of polydactyl expression [Sharpe et al., 1999; Blanc et al., 2002; Lettice et al., 2003; Maas and Fallon, 2005; Sagai et al., 2005]. The spatiotemporal expression of $\mathrm{SHH}$ in the $\mathrm{ZPA}$ is controlled by the long-range cis-regulatory element ZRS, which is located upstream of the Shh gene [Lettice et al., 2002]. Elimination of ZRS elements in mouse results in a limb defect phenotype similar to that in the Shh $\mathrm{Sh}^{-/}$ knockout mouse [Sagai et al., 2005]; it was therefore concluded that the ZRS provides positive regulatory input for SHH expression in the developing limb [Maas et al., 2011]. The expression pattern of SHH is essential for regulating the number and identity of the skeletal elements that compose the digits (toes and fingers) [Yang et al., 1997].

Interestingly, unique point mutations within the ZRS element modify ZRS regulatory effects resulting in various forms of Po in both the human and mouse [Lettice et al., 2003]. Identification of a SNP in strong association with the chicken polydactyl phenotype, found within the coding region of chicken $L M B R 1$, led to the proposal by Huang et al. [2006] that the ZRS element could be the cause of Po in the chicken as mutations in this element result in various forms of Po in human and mouse. Dorshorst et al. [2010] investigated the ZRS in 5 polydactylous chicken breeds and found sequence variation within the 794-bp ZRS, which is located approximately $328 \mathrm{~kb}$ upstream of the $S H H$ gene (GGA2: 8,024,909-8,034,717; www.genome.ucsc.edu). Two of the breeds exhibited a single point mutation (C/A; SNP ss161109890) within a transcription factor binding site known to be involved in limb morphogenesis, while the other 3 breeds did not exhibit that or any other polymorphisms within the 794-bp ZRS. Given these results, it appears that the chicken polydactyl phenotype may result from at least 2 causal mutations; this would be consistent with what is observed in human and other vertebrates [Dorshorst et al., 2010 and references therein]. Recently, Dunn et al. [2011] identified that the ZRS mutation (SNP ss161109890) causes allelic imbalance in heterozygotes with the Po allele preferentially expressed and abnormal regulation of $\mathrm{SHH}$ expression (both prolonged expression and a larger $\mathrm{SHH}$ expression domain when compared to wild-type embryos). This was observed in both posterior and anterior portions of the leg and wing buds during early embryonic development. These changes in SHH expression stimu-

Cytogenet Genome Res 2012;136:50-68 
late the induction of an extra digit on the leg, however, only after specification of digits 1-3 has occurred [Dunn et al., 2011].

Considering the research published to date, it seemed plausible that ZRS is the causative element for the autosomal dominant mutation in UCD-Po.003. To test this hypothesis, the Po.003 genetic line was studied using the ZRS SNP primers established by Dorshorst et al. [2010] to validate the extent to which this single SNP was conserved within the genetic line and segregated with the phenotype. Additionally, the entire UCD-Po.003 ZRS and flanking DNA $(\sim 1 \mathrm{~kb})$ was sequenced to discover additional polymorphisms which could be causative for the phenotypic variation observed within this inbred congenic line. In order to identify whether the polydactyl expression in the UCD-Silkie genetic line is linked to the aforementioned SNP (or a variety of SNPs), the $\sim 1-\mathrm{kb}$ ZRS fragment was also sequenced.

Further, as proposed in Robb et al. [2011], we tested whether a chromosomal inversion was responsible for maintaining the large maximum causative region $\left(\mathrm{CR}_{\max }\right.$ : $6.34 \mathrm{Mb}$ ) using GTG-banding and fluorescence in situ hybridization (FISH) and employing GGA2p BAC and ZRS probes. Lastly, another subset of Po.003 individuals were studied to identify whether any additional recombination events occurred 2 generations after a new breeding scheme was introduced.

\section{Methods}

\section{Genetic Lines}

UCD-Polydactyly.003. The autosomal dominant polydactyl developmental mutation in chicken was obtained by researchers at the University of California during the 1940s. Unfortunately, the origin as to breed and source (research, commercial or hobby stock) of the Po mutant used to generate the UCD-Po.003 genetic line is unknown. In the late 1980s and early 1990s, the mutation was incorporated into a breeding program to create a congenic line using UCD-003, a highly inbred (F > 0.99) Single Comb White Leghorn genetic line [Abplanalp, 1992; Pisenti et al., 1999; Robb et al., 2011]. Here we describe studies conducted utilizing samples from the UCD-Po.003 genetic line to (1) establish whether an inversion within GGA2p is responsible for maintaining the region containing the polydactyl mutation, (2) determine whether the SNP ss161109890 [Dorshorst et al., 2010] is present within the ZRS element, (3) discover additional polymorphisms within the ZRS element to explore the genetic mechanism causing the preaxial polydactyl phenotypic variation, and (4) identify whether additional recombination events reduce the size of the causative (linked) region. During the course of our studies, an extensive evaluation was conducted on the range of the phenotype in the line and included a retrospective historical evaluation of breeding records and progeny phenotypes.
UCD-Silkie. The Silkie, an Asian breed of bantam chicken known for its characteristic dark blue-to-black skin and bones, bright-blue earlobes, and hair-like plumage, was first documented to inhabit regions of China and Japan as early as the 13th century [The American Poultry Association, 1998; Wada et al., 2004]. The UCD-Silkie genetic line was established in 1994 through the donation of 6 exhibition birds acquired from a hobby-breeder in central California [Pisenti et al., 1999]. The UCDSilkie genetic line segregates several mutations: polydactyly $(\mathrm{Po}$, one or more additional preaxial toes), ptilopody ( $P t$, feathered legs and toes), fibromelanosis (Fm, dark pigmented skin and internal organs), crest ( $\mathrm{Cr}$, long feathers on crown of head), muffsand-beard ( $M b$, long feathers under chin), hookless ( $h$, feather defect, no hooklets resulting in hair-like plumage), and the sexlinked inhibitor of dermal melanin (Id, prevents dermal layer pigmentation) [Pisenti et al., 1999]. After establishment of the UCD-Silkie genetic line, birds were initially selected for extreme polydactyl phenotypes with relaxed selection in recent generations.

\section{Sample Collection}

Approximately $0.3 \mathrm{ml}$ of blood was collected in heparin tubes from both UCD-Po.003 ( $\mathrm{n}=11)$ and UCD-Silkie $(\mathrm{n}=14)$ adult birds. From embryos $(\mathrm{n}=11)$ at 10 days of embryogenesis (E10), $0.2 \mathrm{ml}$ of blood was collected by capillary action using microhematocrit capillary tubes (Fisher Scientific) and placed in a $1.5-\mathrm{ml}$ microcentrifuge tube containing $0.4 \mathrm{ml}$ of $0.07 \mathrm{M}$ sodium citrate/ sodium chloride to prevent coagulation. Wing pinfeathers were collected from 79 polydactylous (UCD-Po.003/+) and 30 normal $(+/+)$ (confirmed by phenotyping and genotyping) day-of-hatch chicks and stored in 70\% ethanol. DNA was isolated from the blood and pinfeather samples using the DNeasy Blood \& Tissue Kit (Qiagen) and used for the SNP analyses. For the UCD-Po.003 genetic line, all samples were carefully phenotyped as normal (+/+, 4 toes in a 4-3-2-1 digit conformation) or mutant (heterozygous Po.003/+ or homozygous $P o .003 / P o .003,5$ toes in a $4-3-2-1-2$ ' or 4 toes in a 4-3-2-2' digit conformation; see fig. 1). The number of digits and specific digit conformation was noted for each UCDSilkie adult bird used in this study.

\section{SNP Analysis, Causative Region Identification and ZRS}

\section{Alignment}

The SNP analysis and causative region (CR) identification (maximum and minimum causative region: $\mathrm{CR}_{\max }$ and $\mathrm{CR}_{\min }$, respectively) were defined according to the procedure outlined in Robb et al. [2011]. The SNPs used to fine-map the Po.003 region were selected from the polymorphic Po.003 60K array $\mathrm{CR}_{\max }$ SNPs [Robb et al., 2011]. Additionally, 2 sets of primers for SNP ss161109890 [Dorshorst et al., 2010] were produced for sequencing the 794-bp chicken ZRS element in both genetic lines (UCDPo.003 and UCD-Silkie). Fine-mapping primers are listed in table 1. The ZRS DNA sequences for UCD-Po.003 (NCBI GenBank: JN051261), UCD-Silkie (NCBI GenBank: JN51260), and UCD003 (NCBI GenBank: JN051259) were obtained from sequencing a 1-kb ZRS fragment (794 nt), including 5' (122 nt) and 3' (65 or $66 \mathrm{nt}$ ) sequences (table 1). Human, mouse, and UCD-001 (Red Jungle Fowl inbred line; the sequenced chicken genome, International Chicken Genome Sequencing Consortium [2004]) ZRS DNA sequences were obtained from the UCSC Genome Browser (http://genome.ucsc.edu/). All6ZRS DNA sequences were aligned 

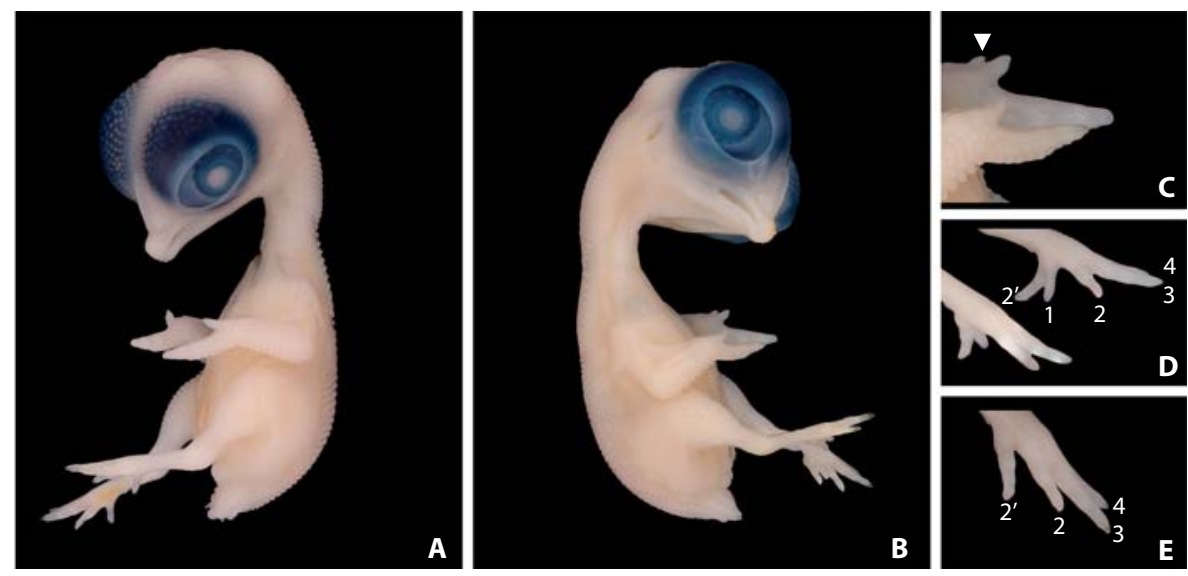

Fig. 1. Variable digit phenotypes of the UCD-Po.003 line. A Normal, non-carrier (+/+) UCD-Po.003 chicken embryo. B UCDPo.003 heterozygote $(P o .003 /+)$ displaying preaxial polydactyly (single-digit duplication) on both feet in a 4-3-2-1-2' digit conformation; preaxial Po evident on the left wing while the right wing is normal. C Enlarged wing from panel B. Preaxial Po displayed only on embryo's left wing (arrowhead). D UCD-Po.003 embryo (Po.003/+) feet displaying preaxial Po in a 4-3-2-1-2' digit conformation; wherein the duplicated digit is 2'. E UCD-Po.003 embryo (Po.003/+) foot displaying preaxial Po in a 4-3-2-2' digit conformation, wherein the elongated digit (additional phalanx on digit 1) is 2'. All individuals shown at 10th day of embryogenesis. using ClustalW (http://www.ebi.ac.uk/Tools/msa/clustalw2/) and visualized in JalView (http://www.jalview.org/) [Thompson et al., 1994; Waterhouse et al., 2009].

Chicken Embryo Fibroblasts: Establishment and Maintenance of Cell Culture

Known (genotyped and test-mating-confirmed) homozygous (Po.003/Po.003) males were mated to normal UCD-003 females $(+/+)$ to obtain heterozygous $(\mathrm{Po} .003 /+)$ progeny for cell culture. Chicken embryo fibroblasts (CEFs) were isolated from E10 Po.003 embryos to create individual-embryo cell cultures [Lima and Macieira-Coelho, 1972]. All polydactylous embryos were phenotyped for morphological characteristics (including gonadal sexing and leg/wing digit pattern) and genotyped using SNP ss161109890, to confirm their heterozygous status. A total of 11 E10 Po.003 CEF cultures were established. Table 2 describes the genetic and phenotypic characteristics of the individual UCDPo.003 cell cultures. Likewise, normal (+/+) UCD-001 CEFs were isolated from E10 embryos and served as a control for investigations regarding GGA2 status. The CEF cultures were grown in DMEM with L-glutamine (Invitrogen), 10\% FBS (Invitrogen), and $1 \%$ penicillin-streptomycin (Invitrogen). At passage 3, chromosome harvests and cytogenetic slides were prepared and stored according to Delany et al. [2007]. Also DT40, a transformed bursa lymphoma cell line [Baba et al., 1985] trisomic for GGA2 [Sonoda et al., 1998; Chang and Delany, 2004; O'Hare and Delany, 2009] was utilized for additional comparison regarding chromosome 2 status.

\section{G-Banding}

G-banding was conducted by modification of the protocol described in Ladjali-Mohammedi et al. [1999]. The UCD-001 CEF and CEF-Po.003 slides were incubated in a $0.25 \%$ trypsin solution $\left(10 \times, 2.5 \%\right.$ stock in $\mathrm{HBSS}$ without $\mathrm{Ca}^{2+}$ or $\mathrm{Mg}^{2+}$; Invit- rogen) for $20 \mathrm{~s}$. Slides were washed briefly (10 s) in HBSS followed by rinsing in Gurr's buffer and then staining for $10 \mathrm{~min}$ in $6 \%$ Gurr's Giemsa stain solution $(\mathrm{pH}=6.8)$. The slides were removed from stain and briefly rinsed (10 s) in Gurr's buffer prior to air-drying.

\section{Probes and Labeling}

Table 3 describes the probes used to investigate the status of GGA2p in the Po.003 line. Specific BACs covering a 10.7-Mb region were identified based upon their GGA2 location using the UCSC Genome Browser (http://genome.ucsc.edu/, Chicken (Gallus gallus) v2.1 (galGal3) assembly, WASHUC2, May 2006; International Chicken Genome Sequencing Consortium [2004]) and obtained from the Children's Hospital Oakland Research Institute (CHORI; http://bacpac.chori.org). Two external anchor BACs (located outside the $\mathrm{CR}_{\max }$ and thus presumed external to a putative inversion) and 1 internal anchor BAC (located in the middle of the $6.34-\mathrm{Mb} \mathrm{CR} \max$ whose position would be unaffected by a putative inversion) were utilized in each FISH experiment. A series of inversion test-BACs were selected to study whether a large portion of the $\mathrm{CR}_{\max }$ was involved in an inversion event. That is, for the test-BACs, if an inversion is present within the GGA2p region of interest on the Po.003 (mutant) chromosome, then the BAC probe locations would be inverted relative to the wild-type chromosome within the Po.003/+ cell or the control samples (CEF UCD-001 and DT40). In addition, BACs were tested that were hypothesized to span the breakpoint of the $\mathrm{CR}_{\text {max }}$, to determine if the probe signals were split producing 2 signals rather than 1 signal on affected (Po.003) versus normal (+) chromosomes (see fig. 2A for the pictorial inversion model). A BAC (CH261-64I4), which includes the ZRS, was utilized to identify the location of the ZRS on GGA2p relative to the other BACs. Similarly, a 958-bp ZRS was amplified from the CH26164I4 BAC using primers listed in table 1. The resulting PCR am- 
Table 1. UCD-Po.003 6-Mb fine-mapping primers

\begin{tabular}{|c|c|c|c|c|c|c|}
\hline SNP ID & $\begin{array}{l}\text { SNP reference } \\
\text { ID }\end{array}$ & $\begin{array}{l}\text { SNP location } \\
(\text { GGA2p) })^{\mathrm{a}}\end{array}$ & Forward primer & Reverse primer & $\begin{array}{l}\text { Melting tem- } \\
\text { perature, }{ }^{\circ} \mathrm{C}\end{array}$ & $\begin{array}{l}\text { PCR frag- } \\
\text { ment size } \\
b p^{b}\end{array}$ \\
\hline Gga_rs15882369 & rs15882369 & $8,012,927$ & CCTGGTTGCCATGAGCTATT & TTCCTTGGGCTTAACATGATCT & 53.5 & 249 \\
\hline Gga_rs15882567 & rs15882567 & $8,082,563$ & CAGCAGGTTGAAGTGATGATG & TTCTGCTGCAAGAGATACCG & 52.5 & 250 \\
\hline Gga_rs14135505 & rs14135505 & $8,104,101$ & CCAAGAAAGGGGATATCTTTCA & TCCCACAACAGTTCATCAGG & 52.5 & 247 \\
\hline Gga_rs14135538 & rs14135538 & $8,135,022$ & CCTCTTCAGCACTGGATTTGA & CTCAGGGCATTTACCAAAGG & 53.5 & 223 \\
\hline Gga_rs14135625 & rs14135625 & $8,273,730$ & GGCATGTGCTGATGCTATGA & GGGAGAGGGCCAGTTGTTAT & 54.0 & 233 \\
\hline Gga_rs14135744 & rs14135744 & $8,366,215$ & CTGCCACTGACTGCTGAAGA & TGGCATCTTAAAGTTGAACCTCT & 53.5 & 231 \\
\hline Gga_rs14135812 & rs14135812 & $8,399,260$ & TTTCCTAGGCTGACGTGCTT & GCTCTTTTCACСТCСАCTGC & 53.5 & 222 \\
\hline $\begin{array}{l}\text { ZRS nested (441 bp) } \\
\text { fragment }\end{array}$ & ss $161109890^{c}$ & $\begin{array}{l}8,414,013- \\
8,414,454\end{array}$ & GCAGTACGCGATTTCCTCTC & AAAAATTTGAGGTAACTTCTTTGC & 53.5 & 441 \\
\hline $\begin{array}{l}\text { ZRS (958 bp) } \\
\text { fragment }\end{array}$ & $\operatorname{ss} 161109890^{c}$ & $\begin{array}{l}8,413,748- \\
8,414,706\end{array}$ & CACCTGCCTTAAGCATACGA & TTTACCATCGTGAGAAACTGG & 51.5 & 958 \\
\hline Gga_rs14135903 & rs14135903 & $8,511,247$ & CCAAATCCCACACTTCTGGT & CTTTGCTTCGCTGTCAGAAA & 53.0 & 249 \\
\hline GGaluGA132991 & & $9,001,066$ & CATTCCCCCTTGTCCTATCA & AGACACATTTTGGGGCTCAC & 53.5 & 292 \\
\hline Gga_rs14137025 & rs14137025 & $9,516,646$ & TTCGGAAAAGCCATTTTCTT & GAAAGTAAAAATACAATATTGCAAACA & 52.5 & 273 \\
\hline Gga_rs14137747 & rs14137747 & $10,066,958$ & GCAAAGGCCTACTTTATCTTTTAGG & GGAACAATCTAGTTCATGCTTTCA & 54.0 & 250 \\
\hline Gga_rs15062381 & rs15062381 & $10,251,785$ & GTTGATGCTTTTGCCAATGA & CCTTTAACAGTGAGTCAAAACCTG & 53.0 & 284 \\
\hline Gga_rs14138100 & rs14138100 & $10,273,547$ & GTGAAGTCATCATGCCCAAG & CCTTTGTATGGTTCCTCTCCA & 52.0 & 243 \\
\hline Gga_rs14138382 & rs14138382 & $10,526,166$ & TGCAATAAATTATACAAAAACAGCA & AAACCACCTGTTTGCTTTCA & 52.0 & 284 \\
\hline Gga_rs14138398 & rs14138398 & $10,559,251$ & GCTGTGGAGAAAATCCTAACCA & AGGTCTTTGCGCTTTTAGCA & 54.0 & 291 \\
\hline GGaluGA133560 & & $11,011,676$ & TGGCAAATGAAATTAAAGCAT & TGGCCACAAGTGTGCTAA & 50.0 & 250 \\
\hline Gga_rs14139117 & rs14139117 & $11,507,344$ & TCCCTAAAAAGCAATGTGAGG & GGTTACGCTTCAAAAATTCCA & 53.0 & 283 \\
\hline Gga_rs14139121 & rs14139121 & $11,527,345$ & CCATGTCCTCCCTACTCAGG & ACAGAACCAGTAGGGCCTCA & 53.0 & 248 \\
\hline GGaluGA133624 & & $11,550,479$ & CAGAAAAGTGGGCATAATCCA & TCATGGTCCTAAACCTGTTGG & 53.5 & 245 \\
\hline Gga_rs15891993 & rs15891993 & $11,578,475$ & TTGTCTGTGAAGGACCGTTG & TGCAGACTGTGTCGAAGACC & 53.0 & 289 \\
\hline Gga_rs14139143 & rs14139143 & $11,584,528$ & GCCCAGCTCAAGTCTTGCT & GGGTTCGCAAGTACAAAGGA & 54.0 & 299 \\
\hline Gga_rs14139194 & rs14139194 & $11,669,333$ & ACACATGGAAGACTTTGAAAATGA & CTTCACCTTTTTGCCCTCAG & 53.5 & 287 \\
\hline Gga_rs14139209 & rs14139209 & $11,694,184$ & GGAAGCATTTTGCTTTCTTGTT & GGAAGAGACAGCTGCCAAAG & 53.5 & 298 \\
\hline Gga_rs15063320 & rs15063320 & $11,756,956$ & AGGCAATCTGGAAGCCATTA & TGAGGAGAAGGTAGAAGACCCTAC & 53.5 & 300 \\
\hline GGaluGA133655 & & $11,759,536$ & GCACAGGCATCAGTTCACAT & ACAGTCTGCACCACTTGCAC & 52.5 & 298 \\
\hline Gga_rs14139263 & rs14139263 & $11,906,712$ & TGACTGTGTTGAATAATAGTGTTTTG & TTTGTGTGTTGACCAAGAATG & 50.5 & 249 \\
\hline Gga_rs14139267 & rs14139267 & $11,926,282$ & TACCTGTGCTGTGTACAATTTTATT & ACACTCACATTTTCCCTTCTGT & 51.5 & 249 \\
\hline Gga_rs14139279 & rs14139279 & $11,958,462$ & TTCAGAAGACTGGAAATTCTTTCA & CCCTTTGCCTTTGGGAAG & 53.5 & 289 \\
\hline Gga_rs14139289 & rs14139289 & $11,989,370$ & GGGGAAGGCAGAACTTGTAG & GGCAGTTATCAACCCGTCTC & 52.5 & 300 \\
\hline Gga_rs15063380 & rs15063380 & $12,017,401$ & GAATACTGGTGGGAAGATTCAA & AGACAACATGAAAAAGACAAGTCA & 52.0 & 249 \\
\hline Gga_rs15892839 & rs15892839 & $12,516,931$ & CAGAACTTAAGAGAATCACAAGCAG & AAGCTGGCTGATTACCCATT & 52.5 & 286 \\
\hline Gga_rs14140258 & rs14140258 & $13,018,274$ & CCAACACAAACATGTGCTTC & GCAGATATGCTTTGCTTCTCTC & 50.5 & 250 \\
\hline Gga_rs14140655 & rs14140655 & $13,517,524$ & AGGAGATGAGGGAGTCAGGAG & ACTGCCTTCATGAGGAGGAA & 53.0 & 238 \\
\hline Gga_rs14140768 & rs14140768 & $13,656,776$ & GGTTGATAGCCCAGTGAGGA & ACGCAGTGGAAAGTTCTTCG & 53.5 & 184 \\
\hline GGaluGA134085 & & $13,813,665$ & CGGCTTTGACACTTTGAACA & CCATAGGGTGAGAGTGTGCAT & 53.0 & 203 \\
\hline Gga_rs13538387 & rs13538387 & $13,948,275$ & TGGCAGCAGATGTTAGAGGA & TTCAAGATGTAAATGGCTCTCG & 53.0 & 200 \\
\hline Gga_rs15896134 & rs15896134 & $13,982,345$ & GCAGTGAATGGATGAAAGCA & CAAGCTGCTCTGAAACCTGA & 52.5 & 212 \\
\hline GGaluGA134145 & & $13,993,610$ & TCATGTTTCTCTTGTTCTGTACCC & AGCCTCCCAGCTTACCGTAT & 53.5 & 209 \\
\hline Gga_rs15896212 & rs15896212 & $14,024,326$ & ACCAGCATTGTTCCCTCATC & ACGCCTCATACTTCCACGTC & 53.5 & 244 \\
\hline
\end{tabular}

${ }^{a}$ Chromosomal location (bp) of SNP on GGA2p; positions are based on the 2006 Gallus gallus assembly (WASHUC2).

${ }^{\mathrm{b}}$ PCR fragment size was determined by 3 methods: (1) using the UCSC Genome Browser (http://genome.ucsc.edu/, 2006 Gallus gallus assembly (WASHUC2)), (2) sizing by gel electrophoresis, and (3) DNA sequencing.

c SNP ss161109890 is the chicken ZRS SNP identified by Dorshorst et al. [2010].

plicon (958 bp) was size-separated on a 1.0\% 1× TAE agarose gel, extracted with the Qiaquick Gel Extraction kit (Qiagen), and sequence-verified (Davis Sequencing, Davis, Calif., USA). The confirmed product was then TA-cloned into the pGEM T-Easy vector (Promega) and sequence-verified again prior to use as a FISH probe.

Approximately $1.5 \mu \mathrm{g}$ of each BAC and/or ZRS clone was labeled either with digoxigenin by the DIG Nick Translation Mix (Roche, Indianapolis, Ind., USA), or with Enzo-Red or Enzo-Orange dUTP by the Nick Translation Kit (Abbott Molecular, Inc., Des Plaines, Ill., USA). Probes labeled with digoxigenin were detected following incubation with fluorescein (FITC) anti-DIG antibody ( $0.70 \mu \mathrm{g} / \mathrm{slide}$; Roche).

\section{FISH and Chromosome Analysis-Imaging}

The multi-color FISH procedures were followed as described in O'Hare and Delany [2009]. Exceptions include the following: slides were heat-treated for $24 \mathrm{~h}$ at $65^{\circ} \mathrm{C}$ in a dry incubator prior to dehydration in 70,80 , and $95 \%$ ethanol; slide preparations were denatured using $70 \%$ deionized formamide at $66^{\circ} \mathrm{C}$ for $55 \mathrm{~s}$; and probes were added to the slide in a mixture containing $10 \mu \mathrm{ldi}$ goxigenin-labeled probe(s), $15 \mu \mathrm{l}$ hybridization mix, and $15 \mu \mathrm{l}$ Enzo-Red or Enzo-Orange-labeled probe(s). Approximately $40-$ 90 cells per experiment were examined utilizing an Olympus BX41 epifluorescence microscope equipped with an automatic filterwheel (Chroma Technology 8200), Spectrum Red filter, X-cite 120 Series metal-halide fiber optic lamp and Applied Imaging CytoVision Genus 3.93 software (Genetix). 


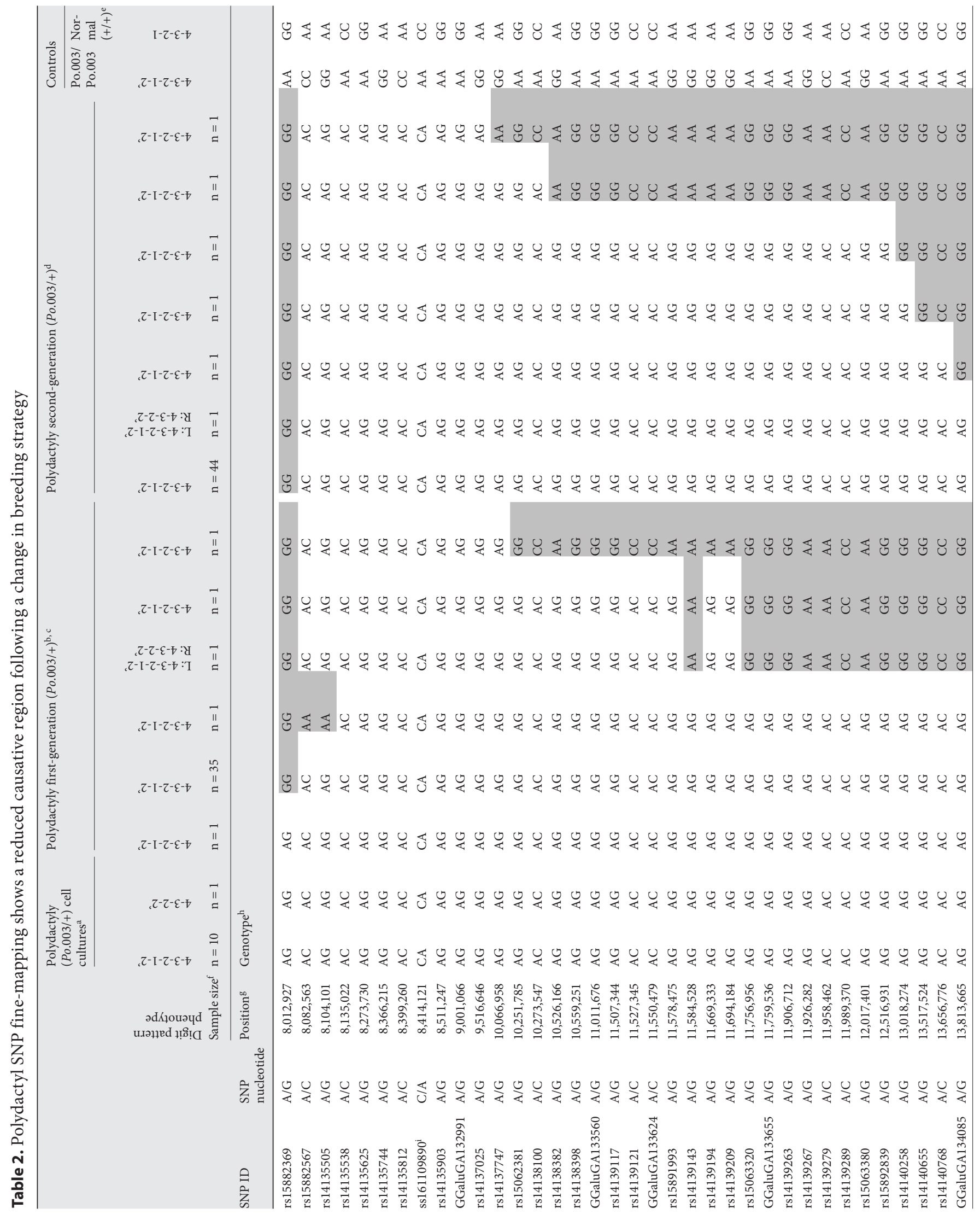




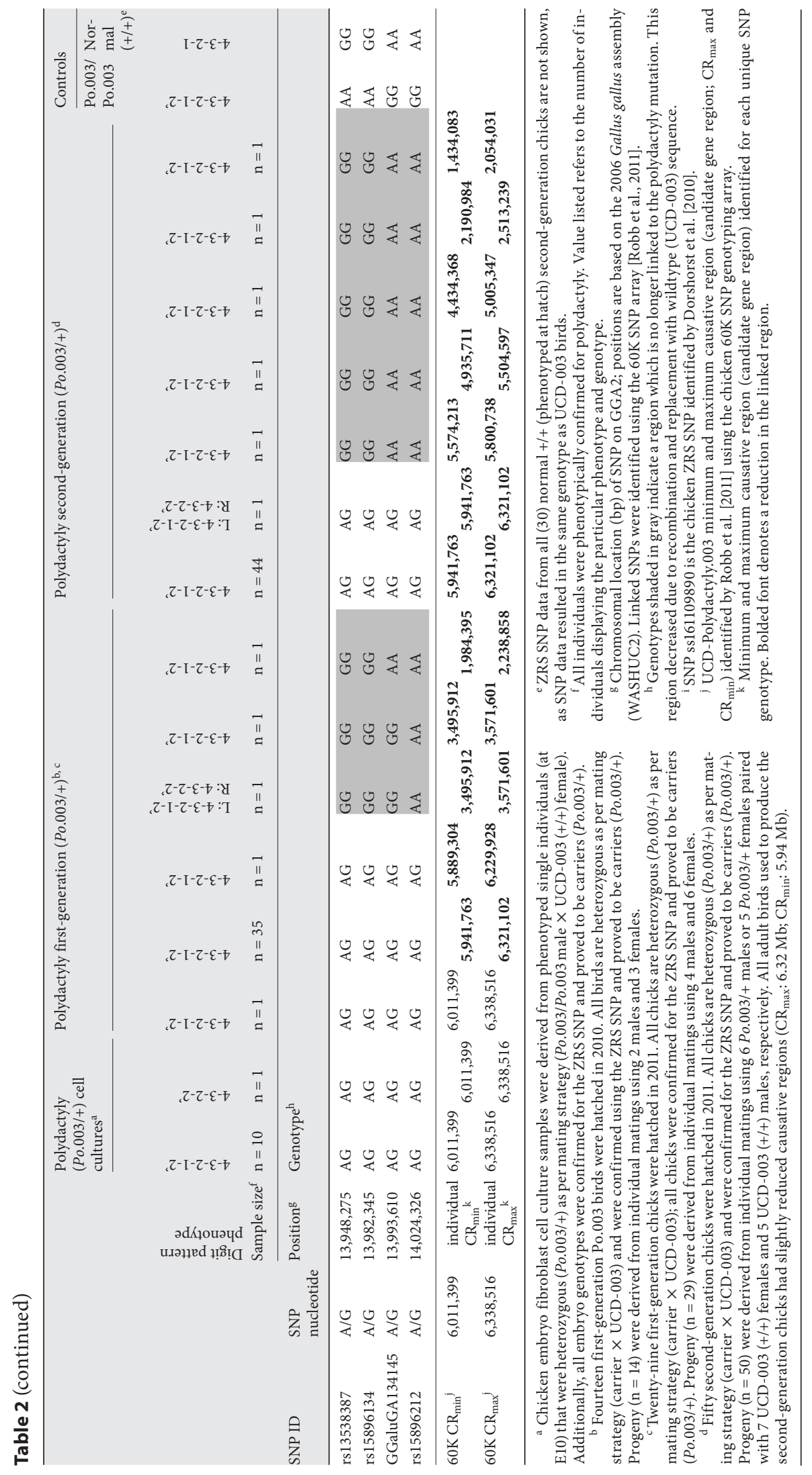


Table 3. Details of chicken chromosome-specific probes

\begin{tabular}{|c|c|c|c|}
\hline Clone identification $^{\mathrm{a}}$ & GGA2 coordinates ${ }^{\mathrm{b}}$ & Insert size, bp & FISH localization $^{c}$ \\
\hline CH261-177B13 & $5,989,880-6,129,780$ & 139,901 & $2 p$ \\
\hline TAM32-13P10 & $7,902,033-8,077,860$ & 175,828 & $2 \mathrm{p}$ \\
\hline $\mathrm{CH} 261-92 \mathrm{~A} 14^{\mathrm{d}}$ & $7,945,672-8,143,627$ & 197,956 & $2 \mathrm{p}$ \\
\hline ZRS & $8,413,748-8,414,706$ & 958 & $2 \mathrm{p}$ \\
\hline $\mathrm{CH} 261-100 \mathrm{E} 23$ & $9,250,689-9,459,587$ & 208,899 & $2 \mathrm{p}$ \\
\hline CH261-90G13 & $11,276,559-11,453,814$ & 177,256 & $2 \mathrm{p}$ \\
\hline $\mathrm{CH} 261-57 \mathrm{O} 16$ & $13,019,233-13,219,485$ & 200,253 & $2 \mathrm{p}$ \\
\hline CH261-32L11 & $16,474,415-16,683,175$ & 208,761 & $2 \mathrm{q}$ \\
\hline
\end{tabular}

a BACs labeled CH261-\# were obtained from the Children's Hospital Oakland Research Institute, CH261 EcoRI BAC library (http://bacpac.chori.org). BAC TAM32-13P10 (Texas A\&M University, TAM32 EcoRI BAC library) was obtained from Dr. Jerry Dodgson at Michigan State University. The ZRS (958-bp insert) DNA plasmid was amplified from BAC CH261-64I4 and subsequently cloned (see Methods).

b The BAC GGA2 coordinates were from http://genome.ucsc. edu/, 2006 Gallus gallus assembly (WASHUC2). GGA2 includes $\sim 154 \mathrm{Mb}$ of genomic sequence: $\mathrm{p}$ arm from 0 to $52.3 \mathrm{Mb}, \mathrm{q}$ arm from 53.8 to $154.9 \mathrm{Mb}$.
${ }^{c}$ FISH localization of BACs or plasmid to chicken chromosomes.

$\mathrm{d}$ The hybridization profile for these 3 BACs indicates a major signal (telomere-proximal) on GGA2p plus a minor signal on $2 \mathrm{q}$ centromere-proximal.

e According to the UCSC Genome Browser, these BACs contain the ZRS element within the LMBR1 intron 5.

${ }^{f}$ BAC CH261-76J5 hybridized to GGA4p, although it was positioned to GGA2p: 8,363,435-8,586,925. This could be due to misassembly, although BAC-end BLAT analysis indicates sequence similarity to GGA2p but not GGA4p. Similarly, hybridization to GGA4p could be simply because the wrong BAC was shipped.

\section{Results and Discussion}

The UCD-Po.003 mutation was developed as a congenic line (see Methods) in anticipation of molecular and genomic tool advancements to allow for mapping and causative element analysis [Pisenti et al., 1999]. A recent SNP-array mapping study described by Robb et al. [2011] established the causative element for the UCD-Po.003 mutation to reside within a 6-Mb region on GGA2p; this result confirms the Po-linked region identified in other chicken breeds [Pitel et al., 2000; Dorshorst et al., 2010; Dunn et al., 2011]. Although this region has been studied in other chicken stocks, knowledge of the details of the phenotype and the causative element for the Po mutation in the UCD-Po.003 line were lacking. Below we describe the phenotypic variation and genetic mechanisms underpinning the UCD-Po.003 mutation. Further, we discuss the results in the context of the Po condition in other chicken breeds and vertebrates.

\section{UCD-Po.003 Phenotypic Variation}

Within the UCD-Po.003 line, polydactyl expression is variable and temperature sensitive. Homozygous
(Po.003/Po.003) and heterozygous (Po.003/+) mutants may have an additional preaxial digit (single-digit duplication) on 1 or both feet [Robb et al., 2011] in a 4-3-2-1-2' digit conformation, wherein the duplicated digit is 2 '. This duplicated digit 1 is longer than the normal digit 1 (short, innermost toe) due to the presence of an extra phalanx (terminal digit bone). This digit pattern is the most common among chicken polydactyl phenotypes [Warren, 1944]. In contrast to the additional digit, polydactylous mutants may also display a longer-than-normal first digit on 1 or both feet in a 4-3-2-2' digit conformation (fig. 1). This form of Po, termed polyphalangy [Warren, 1944], results from the addition of an extra phalanx on the normal digit 1 rather than a duplication of the digit. The digit variation (4-3-2-1-2' or 4-3-2-2') can be expressed on both feet or in a unilateral fashion with 1 form on each foot (heterodactyly), with a preference towards 4-3-2-2' on the bird's right leg; the latter conformation is less common in hatched birds than embryos. These polydactyl foot conformations have also been reported in other chicken breeds [Bond, 1920, 1926; Punnett and Pease, 1929; Sturkie, 1943; Warren, 1944]. Furthermore, any of the foot polydactyl phenotypes mentioned above 

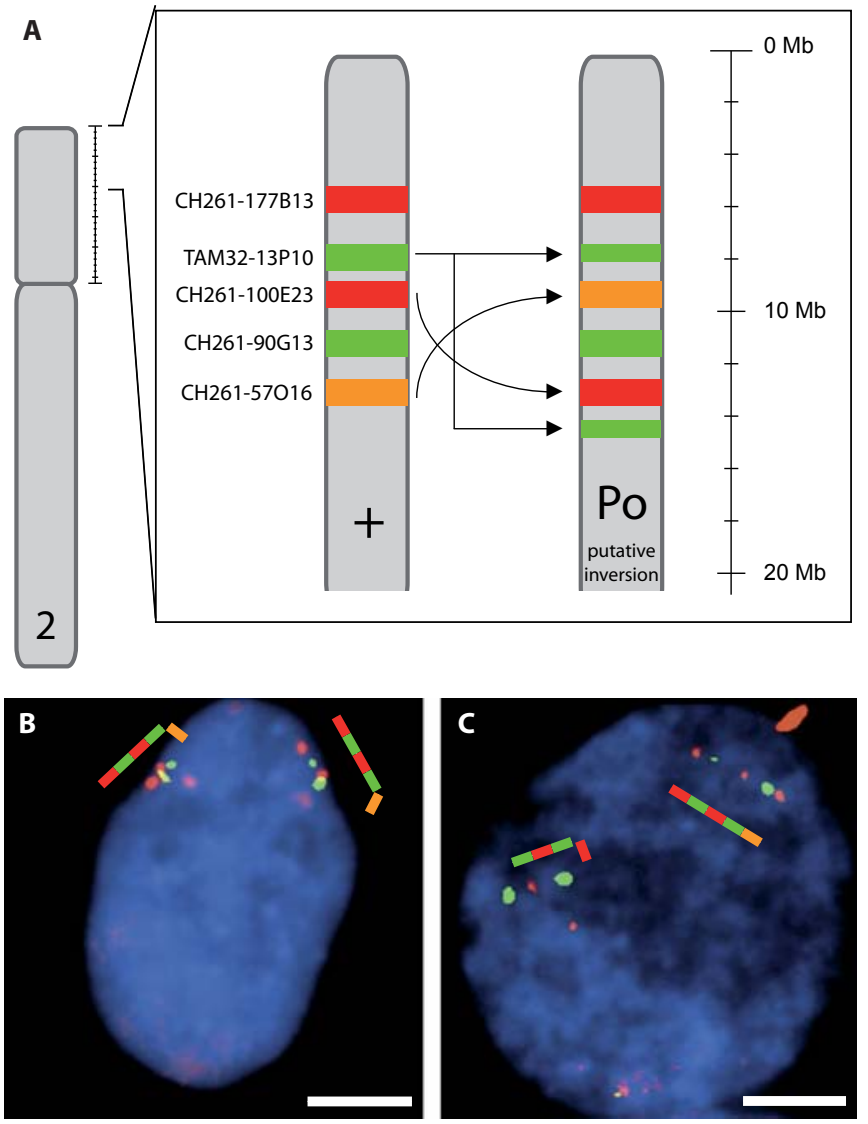

Fig. 2. UCD-Po.003 (Po.003/+) GGA2p inversion model and representative FISH BAC probe pattern from interphase cells. A The enlarged inset illustrates the BAC-probe order (over a 8.4-Mb region) and color profile predicted for both the normal homolog (left) and a predicted inversion for the GGA2p region of Po.003 (right). In the case of the normal homolog $(+)$, the BAC order pattern would be as follows: CH261-177B13 (red), TAM32-13P10 (green), CH261-100E23 (red), CH261-90G13 (green), and CH261$57 \mathrm{O} 16$ (orange). The inset chromosomal image on the right depicts the predicted BAC order profile given an inversion: $\mathrm{CH} 261-$ 177B13 (red), TAM32-13P10 (green), CH261-57O16 (orange), CH261-90G13 (green), CH261-100E23 (red), and TAM32-13P10 (green); note the inversion of BACs CH261-100E23 and CH26157O16, and the TAM32-13P10 split FISH signal. See table 3 for specific BAC coordinates spanning the region of 5-14 $\mathrm{Mb}$ on GGA2p (0-52.3 Mb). B Interphase cell from a normal (+/+) UCD$001 \mathrm{CEF}$ cell line displaying BAC probe order in the noninversion BAC pattern: CH261-177B13 (red), TAM32-13P10 (green), CH261-100E23 (red), CH261-90G13 (green), and CH261-57O16 (orange). C Interphase cell from the UCD-Po.003 CEF heterozygote $($ Po.003/+) displaying BAC probe signaling on both homologs in the normal (noninversion) BAC pattern. If an inversion was present, 1 homolog would display the inversion BAC pattern (see A), while the other chromosome would have the wild-type. Only 1 GGA2 homolog shows the orange probe (CH261-57O16) hybridization signal; however, this is not uncommon given hybridization efficiencies in multicolor FISH. Colored blocks depict the representative probe pattern and chromosome orientation. Scale bar $=5 \mu \mathrm{m}$. can be in addition to 1 or both preaxial polydactyl wing(s). For example, the embryo displayed in figure $1 \mathrm{~B}$ and $\mathrm{C}$ has Po only on the left wing.

Variable penetrance of the polydactylous phenotype has been reported in several chicken breeds and genetic lines [Punnett and Pease, 1929; Pitel et al., 2000; Bouldin and Harfe, 2009], but has not been documented for the UCD-Po.003 congenic line. Herein, we report penetrance to be 1.0 as $100 \%$ of the individuals with the Po.003 allele have some form of polydactyl expression (feet and/or wings). The expressivity of this polydactylous mutation is variable (fig. 1B-E), although not dependent upon the ZRS SNP genotype (see ZRS SNP Identification below).

Interestingly, incubation of UCD-Po.003 embryos at an increased temperature $\left(37.7^{\circ} \mathrm{C}\right.$ rather than the normal $37.3^{\circ} \mathrm{C}$ ) allows for a greater prevalence of the 4-3-2-1-2' digit pattern and preaxial Po on the wing(s), thereby indicating that a temperature-sensitive element is involved. Likewise, decreased incubation temperatures inhibit the expression of a polydactylous phenotype in most breeds [Sturkie, 1943; Warren, 1944 and references therein]. Since UCD-Po.003 individuals share the same inbred genetic background [see Robb et al., 2011 for further description], 1 potential source of the phenotype variability could be the incubation environment (i.e. placement of egg proximal or distal from heat source and/or fan). In order to identify whether the phenotypic variability is due to positioning of the egg relative to the heat source and/or fan, we examined the breeding records for recorded phenotype. Assessment of the polydactyl phenotypes among mutant (Po.003/Po.003 or Po.003/+) chick and adult individuals $(\mathrm{n}=197)$ over a 13-year period (19992011) revealed that $84.26 \%$ of the individuals displayed the 4-3-2-1-2' digit conformation on both feet, while $11.68 \%$ exhibited the 4-3-2-2' digit pattern on both feet; $4.06 \%$ of the 197 individuals displayed the heterodactylous phenotype. An ANOVA of the phenotype incidences showed a statistically significant difference and Tukey's pairwise comparisons $(\alpha<0.001$; using Minitab 15) showed polyphalangy and heterodactyly incidence to be statistically significant from the 4-3-2-1-2' digit conformation. However, the incidences of the polyphalangy and heterodactyly phenotypes were not found to be statistically significant from each other. These results suggest that the phenotypic variability is controlled by either genetic or epigenetic mechanisms or both in concert as polyphalangy and heterodactyly are neither occurring at random nor due to an incubation-temperature effect (as position in the incubator was recorded and indicated no association). 
Table 4. Mendelian inheritance analysis of UCD-Polydactyly.003 phenotypic variation ${ }^{\mathrm{a}}$

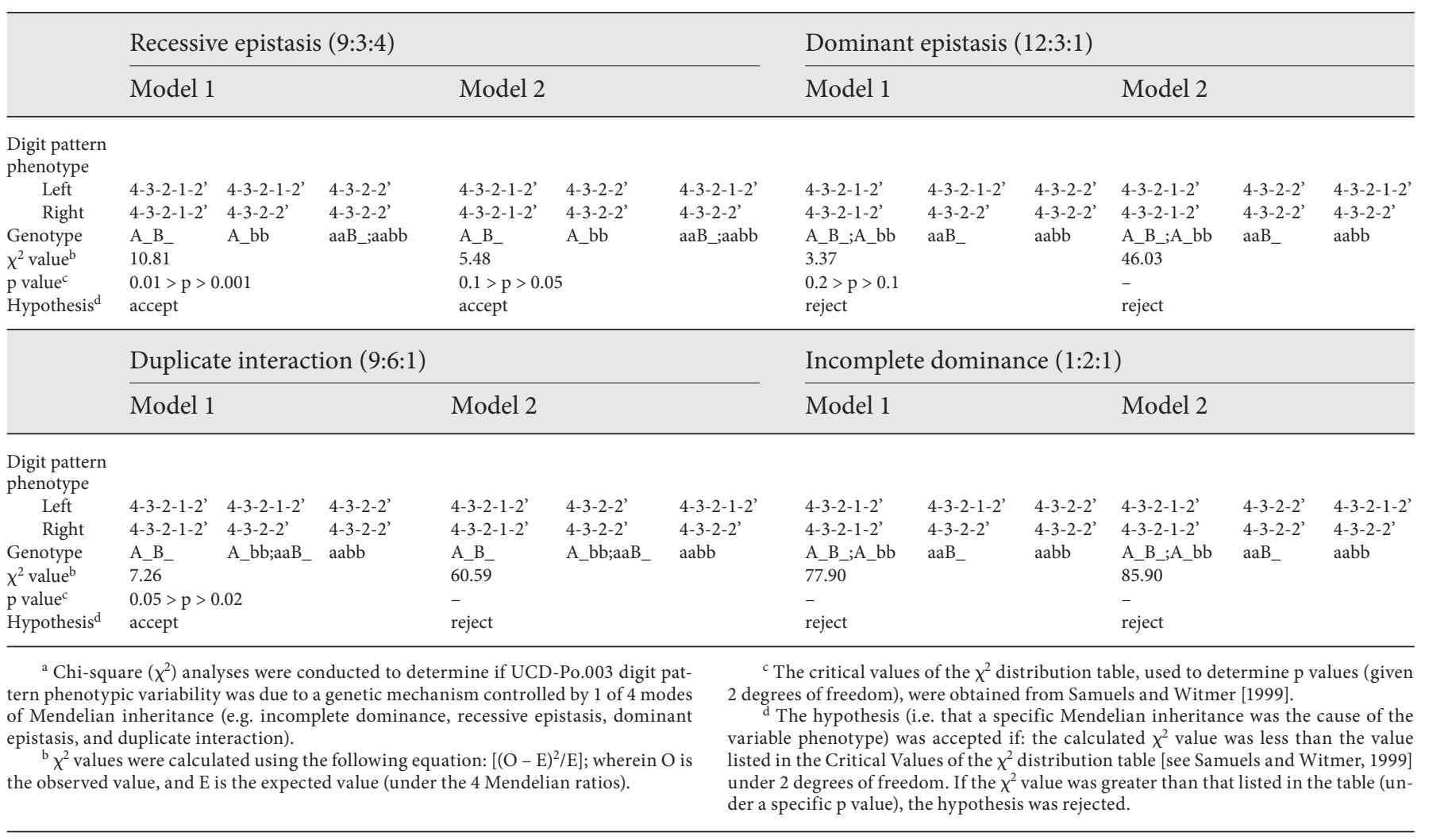

The prevalence of the 4-3-2-1-2' digit pattern, and the polyphalangy and heterodactyly phenotypes, have been noted by numerous researchers dating back 90 years [Bond, 1920 and references therein; Warren, 1944]. To investigate the potential for an underlying genetic mechanism causing the phenotypic variation, we evaluated 4 Mendelian inheritance models (i.e. incomplete dominance, recessive epistasis, dominant epistasis, and duplicate interaction) using a $\chi^{2}$ test (table 4 ). We assessed the phenotypes of 84 mutant (Po.003/+) embryos of known parentage at E14, a stage at which mortality was not an issue. Notably, the grandsire of these embryos was a homozygous (Po.003/Po.003) male mated to several UCD$003(+/+)$ females (F > 0.99) [Abplanalp, 1992; Robb et al., 2011]; resulting progeny (heterozygotes) were mated to UCD-003 and were used as the parents of the 84 polydactyl mutants. The use of 1 Po.003/Po.003 grandsire has the advantage of a simpler genetic assessment of the variation rather than looking at the population level. As seen in table 4, 2 modified dihybrid phenotypic ratios (due to gene interaction) showed significant results $(\mathrm{p}<0.05)$ by $\chi^{2}$ tests. Significant results were found for recessive epis- tasis (under both models; see table 4) and duplicate interaction (under model 1) while inheritance by dominant epistasis and incomplete dominance were ruled out. The ratios for the 2 significant phenotypic inheritance models are 9:3:4 and 9:6:1; wherein the most significant $p$ values are seen using the following categories: 4-3-2-1-2', heterodactyly, and polyphalangy, respectively. Based on this initial analysis, recessive epistasis appears to be the most likely genetic mechanism for the preaxial polydactyl phenotypic variation (see table 4). Considering these results, we propose that at least 2 additional genetic elements outside the ZRS, but within the linked CR $(\sim 6.3$ $\mathrm{Mb}$ ), in addition to the identified ZRS SNP (ss161109890), are the cause of the phenotypic variation seen in the Po.003 genetic line (see ZRS SNP Identification below). A possible candidate for phenotypic variation could be the highly associated T1254C mutation found in LMBR1 exon 16, wherein Huang et al. [2006] hypothesized that this element could act as a functional unit regulating the expression of Po. Further sequence exploration outside the ZRS, but within the linked CR, will be necessary in order to identify the exact elements and mechanism(s) 
underlying the various digit patterns seen in UCDPo.003.

\section{ZRS SNP Identification}

UCD-Po.003. In mouse and human, unique sequence characteristics within the ZRS, including deletions, point mutations, and chromosomal looping [Ianakiev et al., 2001; Maas and Fallon, 2004; Amano et al., 2009] have been shown to cause various forms of Po (e.g. preaxial Po, triphalangeal thumb, polysyndactyly) [Gurnett et al., 2007; Wang et al., 2007; Furniss et al., 2008; Lettice et al., 2008; Sun et al., 2008]. In order to identify whether the UCD-Po.003 mutation was due to 1 or more polymorphisms, as proposed by Dorshorst et al. [2010], in addition to identifying the factor contributing to the digit pattern variation (heterodactyly and/or polyphalangy), a $\sim 1-\mathrm{kb}$ genomic fragment, which included the 794-bp ZRS and flanking DNA, was sequenced.

A total of 90 heterozygous (Po.003/+) birds, 11 heterozygous Po.003 cell cultures (derived from single embryos, see Methods), and 30 normal birds were examined. In all cases, the SNP genotyping results corroborated the phenotypes and the expected genotypes (from specific matings). Thus, SNP ss161109890 is conserved within the UCD-Po.003 genetic line (see table 2) and no other sequence elements varied within the 794-bp ZRS when compared to the genetic background, UCD-003 (+/+) and reference genome UCD-001 (+/+) (fig. 3). This larger data set corroborates results recently reported by Maas et al. [2011], wherein 4 heterozygous UCD-Po.003 individuals were sequenced at the ZRS. Lastly, it is important to note

Fig. 3. ZRS multiple sequence alignment among 4 chicken lines, mouse and human: polydactyl expression is associated with unique SNPs in the ZRS. Comparison of several UCD chicken lines and populations: (1) the congenic UCD-Po.003 genetic line; (2) UCD-Silkie, a breed known for its variety of phenotypes including Po; (3) UCD-001 (inbred Red Jungle Fowl), the sequenced chicken genome; (4) UCD-003 (Single Comb White Leghorn) a highly inbred line (F > 0.99) [Abplanalp, 1992; Robb et al., 2011] and the background genotype for the congenic line UCD-Po.003. The ZRS alignments of the mouse and human are also indicated. The SNP ss161109890 associating with Po in some chicken breeds [Dorshorst et al., 2010] is highlighted in red (position 273; GGA2: 8,414,121; 2006 Gallus gallus assembly (WASHUC2)). Nucleotides highlighted in blue indicate differences among the chicken genetic lines. Nucleotides marked in yellow and green are those that differ in the chicken and mouse, respectively, as compared to human. Gray nucleotides identify bases unique to human. Mutations identified in human and mouse, known to associate with preaxial Po, are bolded and underlined, with a star above the 12 that ss 161109890 is a marker of the polydactylous phenotype and may be the causative mutation in the UCDPo.003 genetic line, but the variation in the expression of the leg and wing preaxial polydactyl phenotype is not correlated with this marker.

UCD-Silkie. A characteristic of the Silkie polydactyl condition is the extreme number of digits ranging from 4-6 per foot [Warren, 1944; The American Poultry Association, 1998]. The breeding scheme of the UCD-Silkie genetic line involved mating individuals with the most extreme Po to each other. Thus, our study aimed to identify if various polymorphisms correlated with specific digit patterns. DNA from 14 UCD-Silkies (4-3-2-1-2', $\mathrm{n}=$ 9; 4-3-2-1-s2', $\mathrm{n}=4$, wherein s2' refers to a split of the 2' digit resulting in 2 distal phalanges and 2 nails, perhaps indicative of delayed start of a 6th digit; and 4'-3-2-1-2', $\mathrm{n}=1$, wherein 4' displays brachydactyly on both feet, i.e. shortened digits) was isolated and the $\sim 800$-bp Silkie ZRS was sequenced and assessed for polymorphisms (table 5). Three SNPs and 1 deletion were discovered relative to UCD-003 (+/+). The sequences for chicken ZRS were deposited in NCBI GenBank: JN051259 (UCD-003 794 nt ZRS plus $122 \mathrm{nt}$ and $66 \mathrm{nt}$ upstream and downstream, respectively); JN51260 (UCD-Silkie 794 nt ZRS plus 122 nt and $65 \mathrm{nt}$ upstream and downstream, respectively); and JN051261 (UCD-Po.003 794 nt ZRS plus $122 \mathrm{nt}$ and $66 \mathrm{nt}$ upstream and downstream, respectively).

The SNP ss161109890 [Dorshorst et al., 2010] was 1 of 3 unique polymorphisms identified in the UCD-Silkie (table 5) and was also recently found in another line of Silkie and shown to result in abnormal SHH expression [Dunn positions [Lettice et al., 2003, 2008; Maas and Fallon, 2005; Sagai et al., 2005; Gurnett et al., 2007; Dorshorst et al., 2010]; for all 12 point mutations, the normal nucleotide is listed. Of note are the number and position of polymorphisms identified among the various chicken genetic lines. SNP ss161109890, identified previously to be associated with the polydactyl phenotype in several lines of chicken has been identified in both UCD-Po.003 and UCD-Silkie (red). Additionally, there are 2 conserved polymorphisms (SNPs) upstream (position -100 (GGA2: 8,413,849) and position -76 (GGA2: 8,413,774)) of the ZRS identified in all Silkie individuals $(\mathrm{n}=14)$ studied (highlighted in blue). Similarly, there is a unique SNP identified in the UCD-003 line at position -67 (GGA2: 8,413,783; highlighted in blue). A deletion event (of a G) was identified in all Silkie individuals at position 565 (GGA2: $8,414,412$; highlighted in blue). Lastly, several insertions were identified but were not incorporated into the alignment as only 1 or 2 UCD-Silkie individuals showed the polymorphism (GGA2: 8,413,765-66 ( $\mathrm{T}$ in 1 bird); GGA2: 8,413,972-73 ( $\mathrm{A}$ or $\mathrm{C}=\mathrm{H}$ in 2 birds); GGA2: 8,413,974-75 (G or $\mathrm{T}=\mathrm{K}$ in 1 bird)) (see table 5).
60

Cytogenet Genome Res 2012;136:50-68
Robb/Delany 
UCD-Po.003 CACCTGCCTTAAGCATACGAACATGTTGCTACAATtTTAAGTĠGTGCTTTTACTAAAATAAATAAAGGAGAATTTGGGAAGAGACATCAAGGAATGACAAAGCAGACGGAGAGCCTAGCTGA UCD-Silkie CACCTGCCTTAAGCATACGAACGTGTTGCTACAATTTTAAGTGGTGTTTTTACTAAAATAAATAAAGGAGAATTTGGGAAGAGACATCAAGGAATGACAAAGCAGACGGAGAGCCTAGCTGA UCD-001 CACCTGCCTTAAGCATACGAACATGTTGCTACAATTTTAAGTGGTGCTTTTACTAAAATAAATAAAGGAGAATTTGGGAAGAGACATCAAGGAATGACAAAGCAGACGGAGAGCCTAGCTGA UCD-003 CACCTGCCTTAAGCATACGAACATGTTGCTACAATTTTAAGTGGTGCTTTTACTAGAATAAATAAAGGAGAATTTGGGAAGAGACATCAAGGAATGACAAAGCAGACGGAGAGCCTAGCTGA CACCTGCCTTAAGCATACGAACATGTTGCTACAATTTTAAGTGGTGCTTTTACTAGAATAAATAAAGGAGAATTTGGGAAGAGACATCAAGGAATGACAAAGCAGACGGAGAGCCTAGCTG $\begin{array}{ll}\text { Mouse } & \text {-A---GCCC-AGGACTTTTT-C-TGGTAGCAG-ATTTATA-TTG-CCCTTTACTTTAATGCCTAT-C-----TTTG--ATTTGAAGTCCTGGCAT-A-AAACT------------TAACATA } \\ \text { Human } & \text {-A----CCC-AAGA-TTTTT-CTGGG-AGTAA-ATTTCTA-TTGT-ACTTTACTTTAATGCCTAT-G------TTTG--ATTTGAAGTCATAGCAT-A-AAAGG------------TAACATA }\end{array}$ $+1$

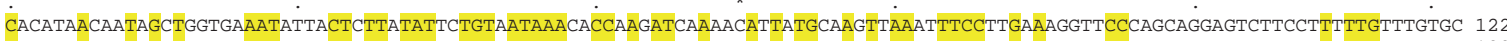
CACATAACAATAGCTGGTGAAATATTACTCTTATATTCTGTAATAAACACCAAGATCAAAACATTATGCAAGTTAAATTTCCTTGAAAGGTTCCCAGCAGGAGTCTTCCTTTTTGTTTGTGC 122 CACATAACAATAGCTGGTGAAATATTACTCTTATATTCTGTAATAAACACCAAGATCAAAACATTATGCAAGTTAAATTTCCTTGAAAGGTTCCCAGCAGGAGTCTTCCTTTTTGTTTGTGC 122 CACATAACAATAGCTGGTGAAATATTACTCTTATATTCTGTAATAAACACCAAGATCAAAACATTATGCAAGTTAAATTTCCTTGAAAGGTTCCCAGCAGGAGTCTTCCTTTTTGTTTGTGC 122 ATGACAGCAACATCC--TGACCAATTATCCAAACCATCCAGCCATCCTAGAG-GTCCAGAACCTCACACATGATCTATAGGATTAAGAGGTTAGCT-----------CCTGTAACTT----- 103

Human 123

AGTACGCGATTTCCTCTCACCCACAAAAAACATACCAAGAATGTGCATGTGCCACTAACACTAAGCAGCACTTCCTTAATCACTCATTTCCAACAATTT-ATGGATGATCAGTG-GCAAAAA 242 AGTACGCGATTTCCTCTCACCCACAAAAAACATACCAAGAATGTGCATGTGCCACTAACACTAAGCAGCACTTCCTTAATCACTCATTTCCAACAATTT-ATGGATGATCAGTG-GCAAAAA 242 AGTACGCGATTTCCTCTCACCCACAAAAAACATACCAAGAATGTGCATGTGCCACTAACACTAAGCAGCACTTCCTTAATCACTCATTTCCAACAATTT-ATGGATGATCAGTG-GCAAAAA 242 AGTACGCGATTTCCTCTCACCCACAAAAAACATACCAAGAATGTGCATGTGCCACTAACACTAAGCAGCACTTCCTTAATCACTCATTTCCAACAATTT-ATGGATGATCAGTG-GCAAAAA 242 - 242

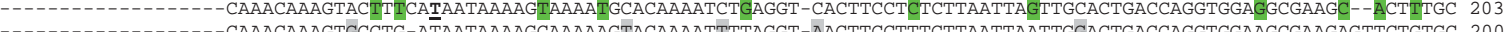
245

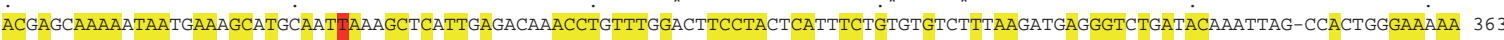
ACGAGCAAAAATAATGAAAGCATGCAATIAAAGCTCATTGAGACAAACCTGTTTGGACTTCCTACTCATTTCTGTGTGTCTTTAAGATGAGGGTCTGATACAAATTAG-CCACTGGGAAAAA 363 ACGAGCAAAAATAATGAAAGCATGCAATGAAAGCTCATTGAGACAAACCTGTTTGGACTTCCTACTCATTTCTGTGTGTCTTTAAGATGAGGGTCTGATACAAATTAG-CCACTGGGAAAAA 363 ACGAGCAAAAATAATGAAAGCATGCAATGAAAGCTCATTGAGACAAACCTGTTTGGACTTCCTACTCATTTCTGTGTGTCTTTAAGATGAGGGTCTGATACAAATTAG-CCACTGGGAAAAA 363 TGG-GCTCAGGCTGT-CCATAAAGCCAAGCAACATGACAGC-ACAATAGAGGAGG-ACTAAGATCGTTTTAATATGTTTCTATCC--TGTGTCACAGTTTGAGATT-GTCC--TGGTTTATG 316 Human 367

ÄGTCATCTGGTCATAAAATACAGTACAGGGTCACTTTTATGTAAgTTTGCCAAAAGGGACATAAACCAGGACAATTTCAAACTGTGACACAGGATAGAAGTATATTAAAAAAATCTTTGTTC 485 AGTCATCTGGTCATAAAATACAGTACAGGGTCACTTTTATGTAAGTTTGCCAAAAGGGACATAAACCAGGACAATTTCAAACTGTGACACAGGATAGAAGTATATTAAAAAAATCTTTGTTC 485 AGTCATCTGGTCATAAAATACAGTACAGGGTCACTTTTATGTAAGTTTGCCAAAAGGGACATAAACCAGGACAATTTCAAACTGTGACACAGGATAGAAGTATATTAAAAAAATCTTTGTTC 485 AGTCATCTGGTCATAAAATACAGTACAGGGTCACTTTTATGTAAGTTTGCCAAAAGGGACATAAACCAGGACAATTTCAAACTGTGACACAGGATAGAAGTATATTAAAAAAATCTTTGTTC 485 TCGCTTTGG-C-DA

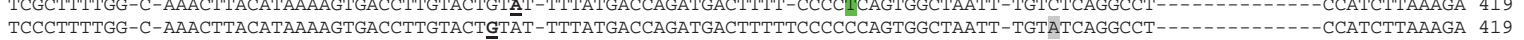

Human 489 513 538 563

588

UCD-P0.003 CंCCTCCATTGTGCTGTCATGTTGCTCAGCTTTATAGACATTCTGAGCACCAGCTCTGGCCTCTTCGCTTCCACCTGGTCTACCTAATTAATTAAGCAAGGAAGTTACCTCAAATTTTTGMA 607 $\mathrm{UCD}-\mathrm{Silkie}$ $\mathrm{UCD}-001$ $\mathrm{UCD}-003$ Mouse Human

CTCCTCCATTGTGCTGTCATGTTGCTCAGCTTTATAGACATTCTGAGCACCAGCTCTGGCCTCTTCGCTTCCACCTGGTCTACCTAATTAATTAAGCAAGGAAGTTACCTCAAATTTTTGTA 607 CTCCTCCATTGTGCTGTCATGTTGCTCAGCTTTATAGACATTCTGAGCACCAGCTCTGGCCTCTTCGCTTCCACCT-GTCTACCTAATTAATTAAGCAAGGAAGTTACCTCAAATTTTTGTA 606
CTCCTCCATTGTGCTGTCATGTTGCTCAGCTTTATAGACATTCTGAGCACCAGCTCTGGCCTCTTCGCTTCCACCTGGTCTACCTAATTAATTAAGCAAGGAAGTTACCTCAAATTTTTGTA 607 CTCCTCCATTGTGCTGTCATGTTGCTCAGCTTTATAGACATTCTGAGCACCAGCTCTGGCCTCTTCGCTTCCACCTGGTCTACCTAATTAATTAAGCAAGGAAGTTACCTCAAATTTTTGTA 60 7

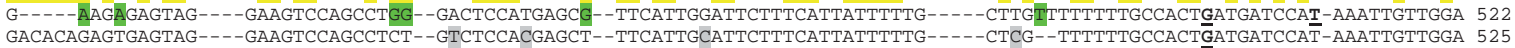
61

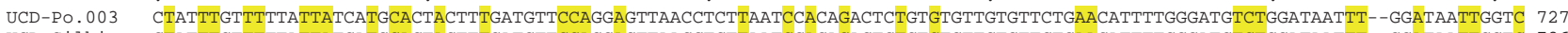
UCD-Silkie CTATTTGTTTTTATTATCATGCACTACTTTGATGTTCCAGGAGTTAACCTCTTAATCCACAGACTCTGTGTGTTGTGTTCTGAACATTTTGGGATGTCTGGATAATTT--GGATAATTGGTC 726 CTATTTGTTTTTATTATCATGCACTACTTTGATGTTCCAGGAGTTAACCTCTTAATCCACAGACTCTGTGTGTTGTGTTCTGAACATTTTGGGATGTCTGGATAATTT--GGATAATTGGTC 727 CTATTTGTITTTATTATCATGCACTACTTIGATGTTCCAGGAGTTAACCTCTTAATCCACAGACTCTGTGTGTTGTGTTCTGAACATTTTGGGATGTCTGGATAATTT--GGATAATTGGTC 727 -AATGAGCGATTCAGG--AAGTGCTGCTTAG-TGTTAGTGGC---------AAATGCGCAAACTCAGTCTG----GTTCTGCT------GGG-TGAAAGGA-AATCACAGGC-AAGAGG-Ä 616 -AATGAGTGATTAAGG--AAGTGCTGCTTAG-TGTTAGTGGC---------ACATGCGCATATTTGGCCTG----GTTCTGGT------GGG-TGAGAGGA-AATCACAGAC-AAAAGG-G 619 733 757 782 807 UCD-Po.003 ÄGATGTTGCTGTCGCTCATGTT---AAACTTTATGCCGTGACTTCGAGTTCAAAGATCAGTATTAAGGT---AAAAT-ACTTCATAAAGCCTTTTTAAAACTCTACACTCCTGATTATGCA 842 UCD-Silkie $\mathrm{UCD}-\mathrm{Silk}$
$\mathrm{UCD}-001$ $\mathrm{UCD}-001$ UCD -003
Mouse AGGATGTTGCTGTCGCTCATGTT---AAACTTTATGCCGTGACTTCGAGTTCAAAGATCAGTATTAAGGT---AAAAT-ACTTCATAAAGCCTTTTTAAAACTCTACACTCCTGATTATGCA 841 AGGATGTTGCTGTCGCTCATGTT---AAACTTTATGCCGTGACTTCGAGTTCAAAGATCAGTATTAAGGT---AAAAT-ACTTCATAAAGCCTTTTTAAAACTCTACACTCCTGATTATGCA 842 AGGATGTTGCTGTCGCTCATGTT---AAACTTTATGCCGTGACTTCGAGTTCAAAGATCAGTATTAAGGT---AAAAT-ACTTCATAAAGCCTTTTTAAAACTCTACACTCCTGATTATGCA 842
AGGCTCCTGCTGGGAACCTTGCAAGGAAATTTGACT---TGGG--CATGTTTTGATCTTGGCATTTATTACAGAAAATGAAGTCATA----------tCT-CACT-----------A 706

Human AAGCCCCTGCTGGGAACCCTGCAAGGAAATTTAACT---TGGGT-CATGTTTTGATCTTAGTGTTTATTACAGAAAATGAAGCCATA---------------TCT-CACT------------A 710 $855 \quad 873$

UCD-P0.003 AंTACCCÄGTT-ATACÄCं 861

UCD-Silkie ATACCCAGTT-ATACCAG 862

UCD-001 ATACCCAGTT-ATACCAG 861

UCD-003 ATACCCAGTT-ATACAGC 861

$\begin{array}{lll}\text { Mouse } & \text { A----CTGTTGCTATGTG } & 720 \\ \text { Human } & \text { A----CTATTGTTACGTG } & 724\end{array}$ 
et al., 2011]. Two additional SNPs were identified upstream of the ZRS element at GGA2: 8,413,849 and 8,413,774 while the deletion (of a $G$ ) is positioned within the ZRS element at GGA2: 8,414,412; these 4 polymorphisms were identified in all Silkie individuals studied. Figure 3 displays the ZRS DNA sequence alignments of 4 UCD chicken genetic lines and as compared to mouse and human. Several insertions were identified but were not incorporated into the alignment as only 1 or 2 UCD-Silkie individuals showed the polymorphism (table 5). None of the SNPs or insertions identified in the UCD-Silkie genetic line were reported in other chicken lines and none match any SNPs previously reported to associate with preaxial Po in both mouse and human [Lettice et al., 2003, 2008; Maas and Fallon, 2005; Sagai et al., 2005; Gurnett et al., 2007; Dorshorst et al., 2010] (fig. 3). Likewise, a particular SNP/ insertion pattern could not be correlated with the various foot-digit phenotypes observed in the UCD-Silkie genetic line. Therein, additional research as to the functionality of each SNP on the polydactyl phenotype would need to be conducted, perhaps using reporter assay experiments similar to that carried out by Maas et al. [2011].

\section{GGA2p Inversion Analysis}

A recent gene mapping study utilizing a $60 \mathrm{~K}$ SNP array with comparison to a previously conducted $3 \mathrm{~K}$ SNP array found that the size of the CR associated with the Po.003 mutation had decreased by only $142 \mathrm{~kb}$ and was maintained at $\sim 6 \mathrm{Mb}$ [Robb et al., 2011]. Given a recombination rate of $1.5-3.5 \mathrm{cM} / \mathrm{Mb}$ in this region [Groenen et al., 2009; Elferink et al., 2010], one would predict at least 9 recombination events to occur within the $\sim 6-\mathrm{Mb}$ $\mathrm{CR}$, thereby reducing the $\mathrm{CR}_{\max }$ over the 4 -year timeframe between the $3 \mathrm{~K}$ and $60 \mathrm{~K}$ SNP studies. A chromosomal inversion event was proposed as the genetic mechanism for these observations. Support for this hypothesis was that inversion events were associated in several cases of human Po [Lodder et al., 2009; Jeong et al., 2010]. Similarly, using the mouse, Krebs et al. [2003] and Niedermaier et al. [2005] induced chromosomal inversions by radiation, which were approximately $1 \mathrm{Mb}$ and $13 \mathrm{~kb}$ away from the Shh gene, respectively. An outcome of the inversions was lower SHH signaling in the limb bud and offspring with the polydactylous condition.

G-banding of chromosomes was initially utilized to investigate the presence of an inversion event on GGA2p. Both CEF UCD-001 (control, +/+) and CEF-Po.003 (heterozygous, Po.003/+) chromosomes were assessed by comparing the banding pattern of GGA2 homologs within and among the cell cultures, and as compared to the standardized GGA2 banding pattern [Ladjali-Mohammedi et al., 1999]. Due to the high condensation of chromosome arm 2p, evidence for an inversion event (or any other chromosomal rearrangement) could not be ruled out. However, results indicated the Po.003 chromosome was normal in size and architecture, i.e. relative sizes of $\mathrm{p}$ and $\mathrm{q}$ arms, and the centromere position.

Multi-color FISH using BAC probes was employed as a more precise method to investigate the hypothesis of an inversion in the Po.003 line. BACs predicted to be unaffected by an inversion event served as external or internal anchors and test-BACs spanning the breakpoint or located at opposite ends of a putative inversion were selected for analysis (see table 3 for a list of specific BACs and online suppl. fig. 1 for an example of the test-BAC FISH experiments; for all supplementary material, see www. karger.com/doi/10.1159/000335005). A model of the expected multi-color FISH BAC signal predictions in both the presence and absence of an inversion is illustrated in figure 2A. For normal (+) chromosomes, the BACs would be in order according to their specified genome coordinates: CH261-177B13, TAM32-13P10, CH261-100E23, CH261-90G13, and CH261-57O16. If an inversion had occurred in the Po.003 (mutant) chromosome, several different FISH configurations were expected. Based upon genome coordinates, 3 BACs (TAM32-13P10, CH261119L22, and CH261-92A14) were positioned at the putative break point; in the presence of an inversion event, these BACs were predicted to show split signals, i.e. signals at 2 locations separated by $\sim 6 \mathrm{Mb}$. Similarly, the inversion BACs (CH261-64I4, CH261-76J5, CH261-100E23, and/or CH261-57O16) would invert, relative to the wildtype order, and be positioned on the opposite side of the internal anchor BAC (CH261-90G13). As the external BACs are anchors, their positioning on the chromosome arm would remain constant and would serve as a profile control. UCD-001 and DT40 chromosomes showed the expected wild-type BAC order (fig. 2A); similarly, analysis of the UCD-Po.003 heterozygous GGA2 homologs (1 wild-type, 1 mutant) exhibited the same profile as UCD001 and DT40, thereby disproving the inversion hypothesis (fig. 2; online suppl. figs. 1, 2).

There were several unexpected BAC hybridization results. Notably BAC CH261-32L11 hybridized interstitially to GGA2q (fig. 4 ) rather than the $2 p$ position indicated by the UCSC Genome Browser (GGA2p: 16,474,415$16,683,175)$. Based upon probe hybridization position (fig. 4B) and BAC-end sequence analysis (fig. 4C), we suggest CH261-32L11 is located at $\sim 110 \mathrm{Mb}$ on GGA2q. Additionally, 3 BACs (TAM32-13P10, CH261-119L22, and 
Table 5. ZRS sequencing of UCD-Silkie adult birds: identification of unique SNPs and indels

\begin{tabular}{|c|c|c|c|c|c|c|c|c|c|c|}
\hline & $\begin{array}{l}\text { Digit pattern phenotype } \\
\text { Sample size }^{\mathrm{d}}\end{array}$ & \multicolumn{8}{|l|}{ UCD-Silkie } & $\frac{\mathrm{UCD}-003^{\mathrm{a}}}{4-3-2-1}$ \\
\hline G/T & $8,413,745$ & GG & $\mathrm{G} / \mathrm{T}$ & GG & GG & GG & GG & $\mathrm{G} / \mathrm{T}$ & GG & TT \\
\hline $\mathrm{T} / \mathrm{C}$ & $8,413,750$ & $\mathrm{CC}$ & $\mathrm{CC}$ & $\mathrm{CC}$ & $\mathrm{CC}$ & $\mathrm{CC}$ & $\mathrm{CC}$ & $\mathrm{CC}$ & $\mathrm{CC}$ & $\mathrm{TT}$ \\
\hline Insertion & $8,413,973$ & $\mathrm{~A} / \mathrm{C}$ & $\mathrm{A} / \mathrm{C}$ & - & - & - & - & - & - & - \\
\hline Insertion & $8,413,974$ & - & - & - & - & $\mathrm{G} / \mathrm{T}$ & - & - & - & - \\
\hline Deletion & $8,414,412$ & - & - & - & - & - & - & - & - & GG \\
\hline G/C & $8,413,978$ & $\mathrm{G} / \mathrm{C}$ & GG & GG & $\mathrm{CC}$ & GG & $\mathrm{G} / \mathrm{C}$ & $\mathrm{G} / \mathrm{C}$ & $\mathrm{G} / \mathrm{C}$ & GG \\
\hline $\mathrm{G} / \mathrm{T}$ & $8,414,121^{\mathrm{h}}$ & TT & $\mathrm{TT}$ & TT & TT & $\mathrm{TT}$ & $\mathrm{TT}$ & TT & TT & GG \\
\hline
\end{tabular}

${ }^{a}$ UCD-003 is a highly inbred (F > 0.99; Abplanalp [1992]; Robb et al. [2011]) Single Comb White Leghorn line which does not exhibit the polydactyl phenotype.

${ }^{b}$ Although all birds have the 4-3-2-1-2' digit patterning on both feet, this individual displays a split toenail (2 toenails) referred to as 4-3-2-1-s2' patterning, wherein s2' refers to a split of the 2' digit, resulting in 2 distal phalanges and 2 nails, perhaps indicative of delayed start of a 6 th digit.

c Although all birds have the 4-3-2-1-2' digit patterning on both feet, this individual displays brachydactyly (shortness of digits) on digit 4 in both feet (4'-3-2-1-2').

${ }^{\mathrm{d}}$ All UCD-Silkie individuals $(\mathrm{n}=14)$ were hatched in 2010. All individuals were phenotypically confirmed for polydactyly. Value listed refers to the number of individuals displaying the particular phenotype and genotype. e The SNP nucleotide is listed (e.g., +/- = C/A) for those polymorphisms identified upon sequencing the UCD-Silkie genetic line. An insertion denotes those positions in which an insertion was identified in the UCDSilkie genetic line.

${ }^{\mathrm{f}}$ Chromosomal location (bp) of SNP on GGA2; positions are based on the 2006 Gallus gallus assembly (WASHUC2). For the case of an insertion, the base position listed is the 5 base (e.g., for $8,413,766$, the insertion is between position 66 and 67 ).

${ }^{\mathrm{g}} \mathrm{A}$ dash indicates the lack of an insertion or deletion at the coordinate listed.

h SNP ss161109890 is the chicken ZRS SNP identified by Dorshorst et al. [2010].
CH261-92A14) with coordinates between 7.90 and 8.14 $\mathrm{Mb}$ on GGA2p (UCSC Genome Browser), hybridized to GGA2p telomere-proximal (major signal) as expected, but also to $2 \mathrm{q}$ centromere-proximal (minor signal) near the CH261-32L11 hybridization (image not shown). Assessment of the region encompassing the major signal (7.90-8.14 Mb) identified 138 repetitive elements (LINEs, LTRs, STRs, and simple and low complexity repeats) with $27 \%$ of the repeats located in the $\mathrm{SHH}$ gene (UCSC Genome Browser: http://genome.ucsc.edu/). In order to identify why the 3 probes (TAM32-13P10, CH261-119L22, and CH261-92A14) hybridized to GGA2q (other than sharing repeat elements between the 2 locations), we first searched for other members of the hedgehog gene family for localization on GGA2q. Results showed a major cluster of this gene family on GGA2p, but not on 2q. Therefore, crosshybridization among the hedgehog gene family is unlikely the cause for the dual signals. As the exact region of the 3 probe hybridization locations on GGA2q is unknown, we performed BLAT (http://genome.ucsc.edu/cgi-bin/ hgBlat) on chicken $\mathrm{SHH}$ to identify any potential alignments to GGA2q. Twenty-six partial alignments to sequences on GGA2q were returned, with all but 1 align- ment (3,000 nt) less than $350 \mathrm{nt}$ in length. Assessment of these partial alignments identified 14 (of 26) to be tandem repeats (AG and AGAA) within the $\mathrm{SHH}$ genomic sequence. These repeats, paired with repetitive elements within the region, are the likely basis for the hybridization of the 3 BACs to GGA2q. It is interesting, however, that these 3 split-signal BAC sequences, although they contain the aforementioned repeats found elsewhere in the genome, only hybridize near BAC CH261-32L11 on GGA2q. A speculative explanation is that the shared BAC signals between $2 p$ and $2 q$ are due to a minor duplication of this genomic region (GGA2p) on 2q.

\section{Reduction of Causative Region Size: Breeding Scheme and Fine-Mapping}

The breeding strategy between 2002 and 2009 employed full sib-pair matings $(P o .003 \times P o .003)$, whereas in 2010 and 2011 (referred to as the first- and secondgeneration post-60K SNP array), Po.003 (Po.003/+) $\times$ UCD-003 (+/+) matings were conducted. This change in the breeding strategy was instituted in order to determine whether the homozygous mating scheme (closed-line breeding) was responsible for maintaining the large 


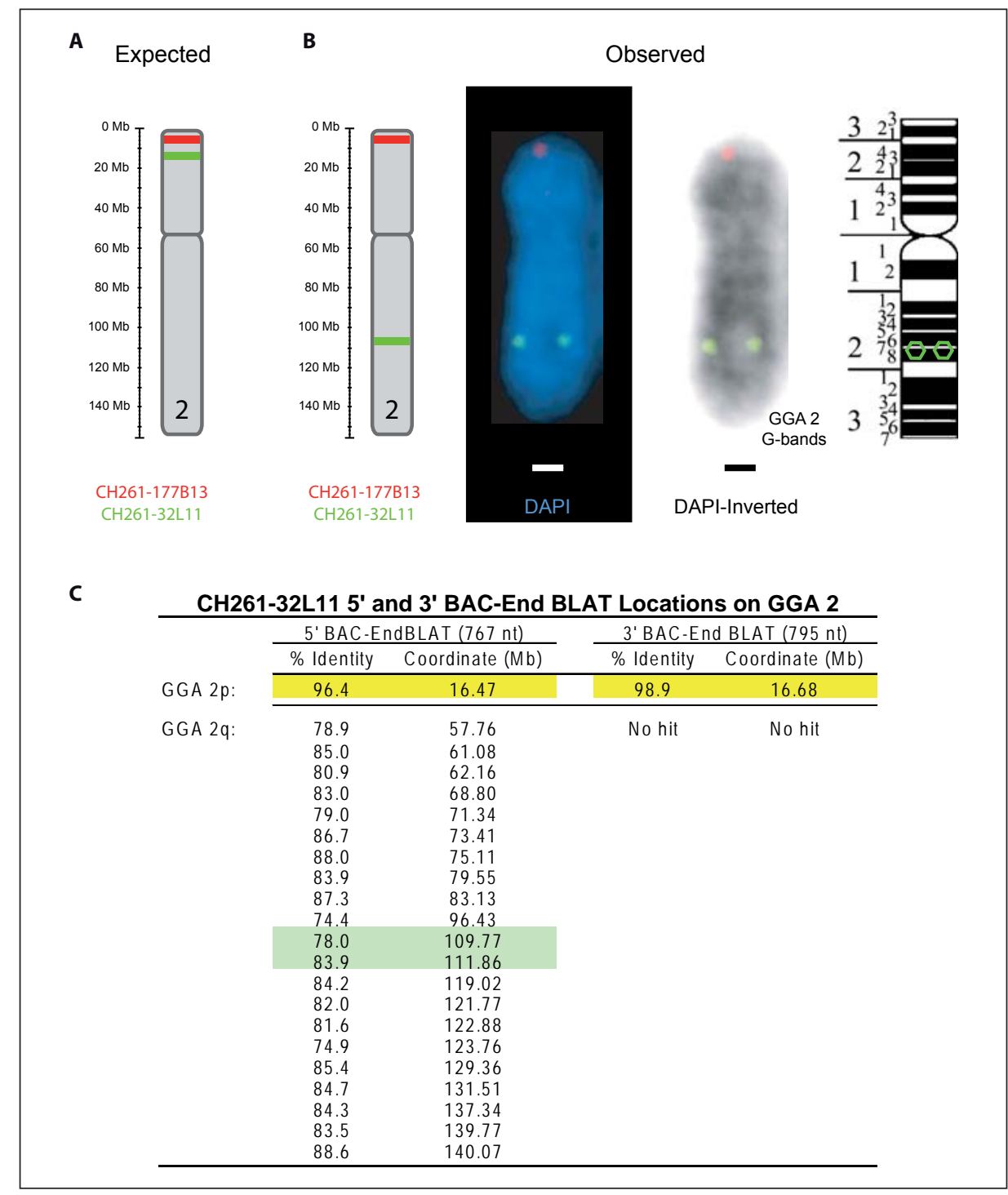

Fig. 4. $\mathrm{BAC} \mathrm{CH} 261-32 \mathrm{~L} 11$ hybridizes to GGA2q rather than GGA2p. Upon cytogenetic analysis, BAC CH261-32L11 hybridized interstitially to GGA2q rather than to the $2 \mathrm{p}$ position indicated by the UCSC Genome Browser (GGA2p: 16,474,415-16,683,175). Scale bar $=1 \mu \mathrm{m}$. A Two external BACs were utilized to initially assess genomic location. BAC CH261-177B13 (red) was expected to be positioned at 5,989,880-6,129,780 (GGA2p) and BAC CH26132L11 (green) was expected to hybridize at 16,474,415-16,683,175 (GGA2p). B Observed FISH results. All 3 cell lines tested (CEF UCD-001, CEF-Po.003, and DT40) showed the same hybridization pattern wherein BAC CH261-177B13 (red) is positioned on GGA2p telomere-proximal, while BAC CH261-32L11 (green) hybridized to GGA2q at an interstitial location. The chromosomal diagram of GGA2 (left) shows the observed location of both probes positioned next to a scale bar. The 2 FISH images (DAPI and DAPI-inverted) show the observed location of the BACs on GGA2p and 2q. Analysis of the inverted DAPI FISH image (inverted results resemble Gbanding) paired with the pictorial G-banded GGA2 (right) [taken from Ladjali-Mohammedi et al., 1999], suggests that BAC CH261$32 \mathrm{~L} 11$ is located on the G-dark band (2q26-28). The green circles on the G-dark band (2q26-28) of the pictorial G-banded GGA2 image indicate the hybridization location of the BAC. Using both the ArkDB database (http://www.thearkdb.org/arkdb/) and the UCSC Genome Browser (http://genome.ucsc.edu), we positioned these G-dark bands to $\sim 110 \mathrm{Mb}$ on GGA2q. C In order to confirm, by sequence assessment, the $\mathrm{CH} 261-32 \mathrm{~L} 11$ hybridization position $(\sim 110 \mathrm{Mb})$ on the G-dark bands $(2 \mathrm{q} 26-28)(\mathbf{B}, \mathbf{C})$, both the $5^{\prime}$ and 3' BAC-ends were BLATed (http://genome.ucsc.edu/cgi-bin/ hgBlat/) against the chicken genome. Reported are the sequence identity and GGA2 coordinate for the sequence results found on GGA2p and 2q. Listed under GGA2p and highlighted in yellow is the position (16.47 and $16.68 \mathrm{Mb}$ ) of the UCSC Genome Browser BAC CH261-32L11 coordinates. Highlighted in green are the sequences located at $\sim 110 \mathrm{Mb}$. Note that the $3^{\prime}$ BAC-end BLAT did not return any sequence identity on GGA2q and the only sequence identity to GGA2p is that listed. Additionally, for the $5^{\prime}$ BAC-end BLAT, 21 other sequence similarities (not listed) were found on GGA2p. GGA2 includes $\sim 154 \mathrm{Mb}$ of genomic sequence, with the $\mathrm{p}$ arm positioned from $0-52.3 \mathrm{Mb}$ and the $\mathrm{q}$ arm from $53.8-154.9$ $\mathrm{Mb}$. 
$\mathrm{CR}_{\max }$ and $\mathrm{CR}_{\min }$ as an alternative to the inversion hypothesis. Genotyping results are presented in table 2 for both generations.

In order to identify a possible reduction in the size of the UCD-Po.003 causative region, the 2010 and 2011 progeny (11 Po.003 CEF cultures (Po.003/+), 11 Po.003 adult birds (Po.003/+), and 79 (Po.003/+) chicks), were assessed at 40 polymorphic SNP locations which included SNPs previously identified as linked to the Po.003 mutation by $60 \mathrm{~K}$ SNP array analysis [Robb et al., 2011] and the ZRS SNP (table 1). As expected, analysis of the Po.003 CEF cultures showed no reduction in the linked CR (table 2). This is because a homozygous (Po.003/Po.003) male $\left(\mathrm{CR}_{\max }: 6.34 \mathrm{Mb}, \mathrm{CR}_{\min }: 6.01 \mathrm{Mb}\right)$ was mated to a normal (+/+) UCD-003 female, producing heterozygous progeny at all loci within the $\mathrm{CR}_{\max }$.

After only 1 generation of backcrossing to UCD-003, 3 progeny indicated a significant reduction for $\mathrm{CR}_{\max }$ and $\mathrm{CR}_{\min }$ to 2.24 and $1.98 \mathrm{Mb}$, respectively (table 2). A second generation of backcrossing, using adults $(n=11)$ from the first-generation backcrossing, led to the reduction of the $\mathrm{CR}_{\max }$ to $2.05 \mathrm{Mb}$ and the $\mathrm{CR}_{\min }$ to $1.43 \mathrm{Mb}$ (table 2). It can be concluded that the closed-line breeding scheme $($ Po.003 $\times$ Po.003), used during the years 2002-2009, maintained the CR associated with the UCD-Po.003 mutation as individuals homozygous for the Po chromosome with large CRs were being mated to each other, thereby preventing a reduction in the linked region. By using a backcross mating scheme for 2 generations, the $\mathrm{CR}_{\max }$ and $\mathrm{CR}_{\text {min }}$ were reduced by 4.28 and $4.58 \mathrm{Mb}$, respectively. In order to maximize the recombination potential, with the goal of further reducing the size of the candidate region and discovering the causative element, one should breed only those individuals that reduced the linked region (maintained a reduced CR relative to others) in subsequent generations. Additionally, it is a decided advantage to have the congenic mutant line (e.g. UCD-Po.003) mate with its inbred parent background line (e.g. UCD-003) in order to further reduce linked regions in congenic lines, especially when small populations are maintained.

Although a reduction in the Po.003 CR was identified after only 2 generations of backcrossing, the breakdown of the region does not support the $1.5-3.5 \mathrm{cM} / \mathrm{Mb}$ recombination rate predicted for this region [Groenen et al., 2009; Elferink et al., 2010]. Of 90 birds (2010, 2011 progeny) only 9 birds exhibited recombination; it can therefore be concluded that the recombination frequency in this region (GGA2p: 8,082,563-14,024,326) for the UCDPo.003 line is slightly lower than predicted. The recombination rate for the linked $\sim 6-\mathrm{Mb}$ region was calculated to be $0.05-0.1 \mathrm{cM} / \mathrm{Mb}(\mathrm{LOD}$ score $(\mathrm{Z})=14.39)$. Since a positive correlation exists between GC content and recombination rates in avian and other mammalian species [Hurst et al., 1999; Williams and Hurst, 2000; Kong et al., 2002; Meunier and Duret, 2004], we assessed the GC content for this $\sim 6-\mathrm{Mb}$ region. Mean GC content for this chromosomal segment is $38.22 \%(\sigma=21.16 \%)$ and was as high as $80 \%$ or more at several locations and as low as $15-35 \%$ in other stretches of the $6 \mathrm{Mb}$ genomic sequence (UCSC Genome Browser: http://genome.ucsc.edu/). Regions of high GC content, observed on average $500 \mathrm{~kb}$ apart, correlate with recombination events identified in the Po.003 individuals; likewise regions of low GC content were associated with regions of no observed crossover events. However, a majority of the high GC content peaks within this genomic region did not have corresponding recombination events, suggesting that some other mechanism could be inhibiting recombination. One possibility concerning the maintenance of this region is that the observed linkage (lack of recombination) is due to double crossover events, thereby resulting in a chromosomal segment that looks like the parental (no recombination). If true, one should see short segments of linked and unlinked regions interspersed throughout the entire $\sim 6-\mathrm{Mb}$ region. An example of this can be seen in the SNP genotype pattern of 2 first-generation individuals (table 2); however, this genotypic pattern is not observed in the other 88 progeny. An alternative explanation is that an unusual chromatin organization (e.g. heterochromatin) in this telomere-proximal region impedes recombination. Interestingly, we found in our FISH experiments that the BACs in this region did not show the expected resolution (separation of signals) (online suppl. fig. 2) given $\sim 2 \mathrm{Mb}$ between each probe, which in other studies was sufficient to achieve separation of signals on extended chromosomes [Laan et al., 1995].

Although the CR has been significantly reduced, further fine-mapping and functional studies will aid in identifying additional genetic elements causing phenotypic variability in the UCD-Po.003 genetic line. Recent studies by Maas et al. [2011] employed the use of a $\beta$-gal reporter assay construct to show that SNP ss161109890 regulates expression of SHH in the Po limb; such a technique could be used to test other polymorphisms for Po causation and phenotype variation. Clearly, the ZRS is important in normal expression of $\mathrm{SHH}$ in the limb, and polymorphisms in this region play a role in the production of Po in many vertebrates. However, more remains to be learned about the genetic mechanisms and developmental pathways involved in digit development and the polydactyl 
chicken model provides great opportunity given the extent of polydactyl genetic resources available, i.e. welldifferentiated polydactylous breeds, closed-line genetic stocks (e.g. UCD-Silkie) and the congenic inbred line UCD-Po.003, which also shows non-random phenotypic variation likely due to an element outside the ZRS.

\section{Conclusions}

Po is a naturally-occurring mutation found in a widerange of vertebrates. In many cases, the cause has been linked to the highly-conserved, non-coding sequence element, ZRS [Maas and Fallon, 2005 and references therein]. The spatiotemporal expression of $\mathrm{SHH}$ in the ZPA is controlled by the ZRS [Lettice et al., 2003] and specific mutations within this conserved sequence influence the polydactyl phenotype [Wieczorek et al., 2010 and references therein]. Our study shows that a single C/A transversion (SNP ss161109890 at coordinate GGA2p: $8,414,121)$, residing within the ZRS element, is associated with the UCD-Po.003 and UCD-Silkie polydactylous phenotypes. Additionally, this SNP can be used as a genotyping marker for determining polydactyl zygosity $(\mathrm{Po} /+$ or $\mathrm{Po} / \mathrm{Po})$ in both lines. Alignment analysis of 4 UCD chicken genetic lines identified 2 novel SNPs, a deletion, and several insertions in the UCD-Silkie line in addition to a unique SNP identified in UCD-003 as compared to the sequenced genome. The variable phenotypes in UCD-Po.003 (2 foot conformations: 4-3-2-1-2' and 4-3$2-2$ ') and UCD-Silkie were not correlated with a particular ZRS SNP pattern; however, based on inheritance analysis we propose that 2 additional modifying elements cause the phenotype variation (e.g. 4-3-2-1-2', heterodactyly, and polyphalangy) in the UCD-Po.003 polydactyl line. The best model for the variation inheritance results is recessive epistasis. The range of polydactyl genetic resources available in the chicken model provides excellent opportunities to explore the molecular and developmental pathways and underlying genetic mechanisms involved in normal and mutant digit formation.

To identify the genetic mechanism maintaining the large 6.34-Mb $\mathrm{CR}_{\max }$ for the UCD-Po.003 congenic line, 2 hypotheses were tested. The first involved a chromosomal rearrangement, the second the breeding strategy. Cytogenetic analysis from both G-banding and FISH showed that UCD-Po.003 maintains 2 normal chromosomes (no inversion event present). A new mating strategy was employed, backcrossing for 2 generations to the inbred parent background line, and the resulting recom- bination events further reduced the size of the CR. Analysis of 40 birds after 1 generation of specialized matings (non-sib pair) showed a reduction in the size of the region identified by Robb et al. [2011] by over $4 \mathrm{Mb}$, to 2.24 and $1.98 \mathrm{Mb}\left(\mathrm{CR}_{\max }\right.$ and $\mathrm{CR}_{\min }$, respectively). Further assessment of 50 chicks ( 2 generations of specialized matings (non-sib pair)) showed a significantly reduced $\mathrm{CR}_{\max }$ and $\mathrm{CR}_{\min }$ (2.05 and $1.43 \mathrm{Mb}$, respectively). It can therefore be concluded that the 6 generations of sib-pair matings and small flock numbers, in addition to a low recombination frequency in the region of interest, maintained the large linked region. Thus, when utilizing forward genetics to identify a gene or region of interest within an inbred-congenic line, it is clear that backcrossing to the background genotype (in this case UCD-003) can lead to further disruption of the maintained region.

\section{Acknowledgements}

We thank Dr. Jackie Pisenti for her dedication to the preservation of the UC Davis poultry genetic resources and for her attention to the breeding and phenotypes of the UCD polydactyl lines. We thank her for her detailed notes on the polydactyly foot digit pattern dating back to 1999 and for her helpful insight regarding this mutation. We also thank Dr. Hans Cheng for helpful conversations regarding this project. Research was supported by the U.S. Department of Agriculture, National Institute of Food and Agriculture Multistate Research Projects NE-1034 (CA-D*-ASC-7281RR), NC-1170 (CA-D*-ASC-6414-RR), NRSP-8 National Animal Genome Research Support Program (CA-D*-ASC-7233-RR) and the University of California, Davis (UCD) John and Joan Fiddyment endowment. We gratefully acknowledge the UCD Department of Animal Science for graduate student fellowship support and the UCD Jastro-Shields Graduate Research Award (EAR). We appreciate the infrastructure and the poultry genetic resources supported by the UCD Department of Animal Science, College of Agricultural and Environmental Sciences, and the California Agricultural Experiment Station.

References

Abplanalp H: Inbred lines as genetic resources of chickens. Poult Sci Rev 4:29-39 (1992).

Amano T, Sagai T, Tanabe H, Mizushina Y, Nakazawa H, Shiroishi T: Chromosomal dynamics at the Shh locus: limb bud-specific differential regulation of competence and active transcription. Dev Cell 16:47-57 (2009).

Baba TW, Giroir BP, Humphries EH: Cell lines derived from avian lymphomas exhibit two distinct phenotypes. Virology 144:139-151 (1985).

-Biesecker LG: Polydactyly: How many disorders and how many genes? 2010 update. Dev Dyn 240:931-942 (2011). 
Blanc I, Bach A, Robert B: Unusual pattern of Sonic hedgehog expression in the polydactylous mouse mutant Hemimelic extra-toes. Int J Dev Biol 46:969-974 (2002).

Bond CJ: On the left-sided incidence of the supernumerary digit in heterodactylous fowls. J Genet 10:87-91 (1920).

-Bond CJ: Further observations on polydactyly and heterodactyly in fowls. J Genet 14:253256 (1926).

-Bouldin CM, Harfe BD: Aberrant FGF signaling, independent of ectopic hedgehog signaling, initiates preaxial polydactyly in Dorking chickens. Dev Biol 334:133-141 (2009).

Chang H, Delany ME: Karyotype stability of the DT40 chicken B cell line: macrochromosome variation and cytogenetic mosaicism. Chromosome Res 12:299-307 (2004).

-Chase HB: Inheritance of polydactyly in the mouse. Genetics 36:697-710 (1951).

-Chiang C, Litingtung Y, Harris MP, Simandl BK, $\mathrm{Li}$ Y, et al: Manifestation of the limb prepattern: limb development in the absence of sonic hedgehog function. Dev Biol 236:421435 (2001)

Danforth CH: Heredity of polydactyly in the cat. J Hered 38:107-112 (1947).

$\checkmark$ Delany ME, Gessaro TM, Rodrigue KL, Daniels LM: Chromosomal mapping of chicken mega-telomere arrays to GGA9, 16, 28 and W using a cytogenomic approach. Cytogenet Genome Res 117:54-63 (2007).

Dennis SM, Leipold HW: Ovine congenital defects. Vet Bull 49:233-239 (1979).

-Dorshorst B, Okimoto R, Ashwell C: Genomic regions associated with dermal hyperpigmentation, polydactyly and other morphological traits in the Silkie chicken. J Hered 101:339-350 (2010).

-Dunn IC, Paton IR, Clelland AK, Sebastian S, Johnson EJ, et al: The chicken polydactyly (Po) locus causes allelic imbalance and ectopic expression of Shh during limb development. Dev Dyn 240:1163-1172 (2011).

-Elferink MG, van As P, Veenendaal T, Crooijmans RP, Groenen MA: Regional differences in recombination hotspots between two chicken populations. BMC Genet 11:11 (2010).

Everman DB: Hands and feet, in Stevenson RE, Hall JG (eds): Human Malformations and Related Anomalies, 2nd ed., pp 935-996 (Oxford University Press, Oxford 2006).

- Furniss D, Lettice LA, Taylor IB, Critchley PS, Giele $\mathrm{H}$, et al: A variant in the sonic hedgehog regulatory sequence (ZRS) is associated with triphalangeal thumb and deregulates expression in the developing limb. Hum Mol Genet 17:2417-2423 (2008).

Gorbach D, Mote B, Totir L, Fernando R, Rothschild M: Polydactyl inheritance in the pig. J Hered 101:469-475 (2010).

-Groenen MA, Wahlberg P, Foglio M, Cheng HH, Megens HJ, et al: A high-density SNP-based linkage map of the chicken genome reveals sequence features correlated with recombination rate. Genome Res 19:510-519 (2009).
Gurnett CA, Bowcock AM, Dietz FR, Morcuende JA, Murray JC, Dobbs MB: Two novel point mutations in the long-range $\mathrm{SHH}$ enhancer in three families with triphalangeal thumb and preaxial polydactyly. Am J Med Genet A 143:27-32 (2007).

-Huang YQ, Deng XM, Du ZQ, Qiu X, Du X, et al: Single nucleotide polymorphisms in the chicken Lmbrl gene are associated with chicken polydactyly. Gene 374:10-18 (2006).

Hurst LD, Brunton CF, Smith NG: Small introns tend to occur in GC-rich regions in some but not all vertebrates. Trends Genet 15:437-439 (1999).

Ianakiev P, van Baren MJ, Daly M, Toledo S, Cavalcanti MG, et al: Acheiropodia is caused by a genomic deletion in C7orf2, the human orthologue of the Lmbrl gene. Am J Hum Genet 68:38-45 (2001).

International Chicken Genome Sequencing Consortium (ICGSC): Sequence and comparative analysis of the chicken genome provide unique perspectives on vertebrate evolution. Nature 432:695-716 (2004).

-Jeong SY, Kim BY, Yu JE: De novo pericentric inversion of chromosome 9 in congenital anomaly. Yonsei Med J 51:775-780 (2010).

Johnson JL, Leipold HW, Schalles RR: Hereditary polydactyly in Simmental cattle. J Hered 72:205-208 (1981).

Kong A, Gudbjartsson DF, Jonsdottir GM, Gudjonsson SA, Richardsson B, et al: A high resolution recombination map of the human genome. Nat Genet 31:241-247 (2002).

Kraus P, Fraidenraich D, Loomis CA: Some distal limb structures develop in mice lacking Sonic hedgehog signaling. Mech Dev 100: 45-58 (2001).

Krebs O, Schreiner CM, Scott WJ, Bell SM, Robbins DJ, et al: Replicated anterior zeugopod (raz): a polydactylous mouse mutant with lowered Shh signaling in the limb bud. Development 130:6037-6047 (2003).

Kren V: Genetics of the polydactyly-luxate syndrome in the Norway rat, Rattus norvegicus. Acta Univ Carol Med Monogr 68:1-103 (1975).

Laan M, Kallioniemi OP, Hellsten E, Alitalo K, Peltonen L, Palotie A: Mechanically stretched chromosomes as targets for high-resolution FISH mapping. Genome Res 5:13-20 (1995).

Ladjali-Mohammedi K, Bitgood JJ, Tixier-Boichard M, Ponce de Leon FA: International System for Standardized Avian karyotypes (ISSAK): standardized banded karyotypes of the domestic fowl (Gallus domesticus). Cytogenet Cell Genet 86:271-276 (1999).

Lettice LA, Horikoshi T, Heaney SJ, van Baren MJ, van der Linde HC, et al: Disruption of a long-range cis-acting regulator for Shh causes preaxial polydactyly. Proc Natl Acad Sci USA 99:7548-7553 (2002).

Lettice LA, Heaney SJ, Purdie LA, Li L, de Beer $\mathrm{P}$, et al: A long-range Shh enhancer regulates expression in the developing limb and fin and is associated with preaxial polydactyly. Hum Mol Genet 12:1725-1735 (2003).
Lettice LA, Hill AE, Devenney PS, Hill RE: Point mutations in a distant sonic hedgehog cisregulator generate a variable regulatory output responsible for preaxial polydactyly. Hum Mol Genet 17:978-985 (2008).

Lima L, Macieira-Coelho A: Parameters of aging in chicken embryo fibroblasts cultivated in vitro. Exp Cell Res 70:279-284 (1972).

Lodder EM, Eussen BH, van Hassel DA, Hoogeboom AJ, Poddighe PJ, et al: Implication of long-distance regulation of the HOXA cluster in a patient with postaxial polydactyly. Chromosome Res 17:737-744 (2009).

Maas SA, Fallon JF: Isolation of the chicken Lmbrl coding sequence and characterization of its role during chick limb development. Dev Dyn 229:520-528 (2004).

-Maas SA, Fallon JF: Single base pair change in the long-range Sonic hedgehog limb-specific enhancer is a genetic basis for preaxial polydactyly. Dev Dyn 232:345-348 (2005).

-Maas SA, Suzuki T, Fallon JF: Identification of spontaneous mutations within the longrange limb-specific sonic hedgehog enhancer (ZRS) that alter sonic hedgehog expression in the chicken limb mutants oligozeugodactyly and Silkie breed. Dev Dyn 240: 1212-1222 (2011).

-Meunier J, Duret L: Recombination drives the evolution of GC-content in the human genome. Mol Biol Evol 21:984-990 (2004).

- Niedermaier M, Schwabe GC, Fees S, Helmrich A, Brieske N, et al: An inversion involving the mouse Shh locus results in brachydactyly through dysregulation of Shh expression. J Clin Invest 115:900-909 (2005).

O'Hare TH, Delany ME: Genetic variation exists for telomeric array organization within and among the genomes of normal, immortalized, and transformed chicken systems. Chromosome Res 17:947-964 (2009).

-Oro AE, Higgins KM, Hu Z, Bonifas JM, Epstein EH, Scott MP: Basal cell carcinomas in mice overexpressing Sonic hedgehog. Science 276: 817-821 (1997).

Park K, Kang J, Subedi KP, Ha JH, Park C: Canine polydactyl mutations with heterogeneous origin in the conserved intronic sequence of LMBR1. Genetics 179:2163-2172 (2008).

Pisenti JM, Delany ME, Taylor RL, Abbott UK, Abplanalp H, et al: Avian genetic resources at risk. An assessment and proposal for conservation of genetic stocks in the USA and Canada. Davis (CA): University of California Division of Agriculture and Natural Resources, Genetic Resources Conservation Program. http://grcp.ucdavis.edu/publications/index.htm. Available from: Report No. 20 (1999).

- Pitel F, Berge R, Coquerelle G, Crooijmans RP, Groenen MA, et al: Mapping the naked neck (NA) and polydactyly (PO) mutants of the chicken with microsatellite molecular markers. Genet Sel Evol 32:73-86 (2000).

- Punnett RC, Pease MS: Notes on polydactyly. J Genet 21:341-366 (1929). 
Riddle RD, Johnson RL, Laufer E, Tabin C: Sonic hedgehog mediates the polarizing activity of the ZPA. Cell 75:1401-1416 (1993).

- Robb EA, Gitter CL, Cheng HH, Delany ME: Chromosomal mapping and candidate gene discovery of chicken developmental mutants and genome-wide variation analysis of MHC congenics. J Hered 102:141-156 (2011).

-Sagai T, Hosoya M, Mizushina Y, Tamura M, Shiroishi T: Elimination of a long-range cisregulatory module causes complete loss of limb-specific Shh expression and truncation of the mouse limb. Development 132:797803 (2005).

-Sakai WH: Polydactyly in a Vaux's swift. Wilson J Ornithol 118:424-426 (2006).

Samuels ML, Witmer JW: Statistics for the Life Sciences, 2nd ed. (Prentice Hall, Englewood Cliffs, 1999).

-Sharpe J, Lettice L, Heckser-Sorensen J, Fox M, Hill R, Krumlauf R: Identification of Sonic hedgehog as a candidate gene responsible for the polydactylous mouse mutant Sasquatch. Curr Biol 9:97-100 (1999).

Sonoda E, Sasaki MS, Buerstedde J-M, Bezzubova O, Shinohara A, et al: Rad51 deficient vertebrate cells accumulate chromosomal breaks prior to cell death. EMBO J 17:598608 (1998).
Struthers J: On the development of the bones of the foot of the horse, and of digital bones generally and on a case of polydactyly in the horse. J Anat Physiol 28:51-62 (1893).

Sturkie PD: Suppression of polydactyly in the domestic fowl by low temperature. J Exp Zool 93:325-346 (1943).

- Sun M, Ma F, Zeng X, Liu Q, Zhao XL, et al: Triphalangeal thumb-polysyndactyly syndrome and syndactyly type IV are caused by genomic duplications involving the long range, limb-specific $\mathrm{SHH}$ enhancer. J Med Genet 45:589-595 (2008).

The American Poultry Association. The American Standard of Perfection (American Poultry Association, Inc., Mendon, MA, 1998).

Thompson JD, Higgins DG, Gibson TJ: CLUSTAL W: improving the sensitivity of progressive multiple sequence alignment through sequence weighting, position specific gap penalties and weight matrix choice. Nucleic Acids Res 22:4673-4680 (1994).

Uehlinger V: A recessive mutation (pd) determinant of polydactyly in Xenopus laevis D. (Batraciens anoures). [French]. J Embryol Exp Morphol 21:207-218 (1969).

Wada Y, Yamada Y, Nishibori M, Yasue H: Complete nucleotide sequence of mitochondrial genome in Silkie fowl (Gallus gallus var. domesticus). J Poult Sci 41:76-82 (2004).
-Wang ZQ, Tian SH, Shi YZ, Zhou PT, Wang ZY, et al: A single $C$ to $T$ transition in intron 5 of $L M B R 1$ gene is associated with triphalangeal thumb-polysyndactyly syndrome in a Chinese family. Biochem Biophys Res Commun 355:312-317 (2007)

-Warren DC: Inheritance of polydactylism in the fowl. Genetics 29:217-231 (1944).

Waterhouse AM, Procter JB, Martin DM, Clamp M, Barton GJ: Jalview Version 2 - a multiple sequence alignment editor and analysis workbench. Bioinformatics 25:1189-1191 (2009).

-Wieczorek D, Pawlik B, Li Y, Akarsu NA, Caliebe A, et al: A specific mutation in the distant sonic hedgehog cis-regulator (ZRS) causes Werner mesomelic syndrome while complete ZRS duplications underlie Haas type polysyndactyly and preaxial polydactyly with or without triphalangeal thumb. Hum Mutat 31:81-89 (2010).

Williams EJ, Hurst LD: The proteins of linked genes evolve at similar rates. Nature 407: 900-903 (2000).

Yang Y, Drossopoulou G, Chuang PT, Duprez D, Marti E, et al: Relationship between dose, distance and time in Sonic Hedgehog-mediated regulation of anteroposterior polarity in the chick limb. Development 124:4393-4404 (1997). 